\title{
A CATALOG OF POINT SOURCES TOWARD NGC 1333
}

\author{
L. M. REBULL \\ Infrared Science Archive (IRSA) and Spitzer Science Center (SSC), Infrared Processing and Analysis Center (IPAC), 1200 E. California Blvd., California Institute of \\ Technology, Pasadena, CA 91125, USA; rebull@ipac.caltech.edu \\ Received 2015 February 6; accepted 2015 April 28; published 2015 June 24
}

\begin{abstract}
I present a catalog of point-source objects toward NGC 1333, resolving a wide variety of confusion about source names (and occasionally positions) in the literature. I incorporate data from optical to radio wavelengths, but focus most of the effort on being complete and accurate from $J(1.25 \mu \mathrm{m})$ to $24 \mu \mathrm{m}$. The catalog encompasses $52^{\circ}<$ R.A. $<52^{\circ} .5$ and $31^{\circ}<$ decl. $<31^{\circ} .6$. Cross-identifications include those from more than 25 papers and catalogs from 1994 to 2014, primarily those in wide use as origins of nomenclature. Gaps in our knowledge are identified, with the most important being a lack of spectroscopy for spectral types or even confirmation of youth and/or cluster membership. I fit a slope to the spectral energy distribution (SED) between 2 and $24 \mu \mathrm{m}$ for the members (and candidate members) to obtain an SED classification, and I compare the resulting classes to those for the same sources in the literature, and for an SED fit between 2 and $8 \mu \mathrm{m}$. While there are certainly differences, for the majority of the sources, there is good agreement.
\end{abstract}

Key words: catalogs - stars: pre-main sequence - stars: protostars

Supporting material: machine-readable and VO tables

\section{INTRODUCTION}

NGC 1333 is one of the youngest and most well-studied starforming regions, in part because it is located at only $\sim 235 \mathrm{pc}$ (Hirota et al. 2008, 2011). Its stars are thought to have an average age of 1-2 Myr (e.g., Bally et al. 2008), but it also contains several Class 0 objects, objects in the earliest stages of star formation (see, e.g., Sandell \& Knee 2001; Sadavoy et al. 2014).

Because this region is very young and relatively nearby, it has been a subject of intensive study for decades. However, there has not yet been a published, comprehensive merging of all of the large catalogs in this region. A summary including each of the prior investigations of NGC 1333 is beyond the scope of this paper; see Walawender et al. (2008) for a recent review. In this paper, my primary goal is to merge all of the available data from the (relatively) large-field surveys in this region and assemble one master catalog with all of the names from the various surveys reconciled. We are studying NGC 1333 as part of the Young Stellar Object VARiability (YSOVAR) project (Rebull et al. 2014, hereafter R14); this catalog was assembled originally as part of that effort. Because my original goal in taking on this task was to focus on the sources for which we have light curves in YSOVAR, some of the data reduction relevant to this paper is described in the YSOVAR overview paper (R14). Some of the detailed investigation described in the present paper is focused on the region mapped by YSOVAR; the region mapped by YSOVAR is the heart of the cluster. The NGC 1333 YSOVAR data are discussed in detail in L. M. Rebull et al. (2015, in preparation).

The catalog is somewhat artificially limited to being within $52^{\circ}<$ R.A. $<52^{\circ} .5$ and $31^{\circ}<$ decl. $<31^{\circ}$. 6 . Data are available over a larger region for some surveys, but this region should include most of the objects actually belonging to NGC 1333, and it entirely includes the region monitored for YSOVAR, as per the original goals in assembling this catalog.

The primary reason I compiled data from several different sources was in order to assemble spectral energy distributions
(SEDs). While, of course, many of the targets vary significantly with time, single-epoch archival data can help define the SED such that, in some cases, the assembled SED can reveal the underlying nature of the source, or at least help narrow the possibilities for the nature of the source.

In this paper, I first review the large surveys that I included in my catalog (Section 2) and then list the papers from which I drew data and explain why I associated or dissociated (or removed) sources across wavelengths (Section 3). I describe obvious gaps in the literature (Section 4). I place the young stellar object (YSO) candidates into SED classes based on the slope between 2 and $24 \mu \mathrm{m}$ and compare them to other schemes from the literature (Section 5).

\section{LARGE DATA SETS}

In this section, I discuss the largest area surveys I used; they are listed, along with the smaller catalogs from the next section, in Table 1.

All of the large-area catalogs described here were merged by position with a catalog-dependent search radius (usually $\sim 1^{\prime \prime}$ ). Many sources, especially those in regions where many sources are close together on the sky, were individually inspected and matched by hand. SEDs were constructed as an additional check on the source matching; objects with particularly strange initial SEDs were also individually inspected and matched by hand, which often resolved any SED issues.

\subsection{MASS and 2MASS $6 \times$}

The Two Micron All Sky Survey (2MASS; Skrutskie et al. 2006) catalog provided the original nucleus of the catalog, to which all other catalogs were merged by position, typically with a $\sim 1^{\prime \prime}$ search radius.

NGC 1333 was in the original 2MASS, of course, and it was also included in the long-exposure $6 \times 2$ MASS program. The original 2MASS data were obtained in 2000 . I included the main 2MASS catalog and the deeper $6 \times$ catalog near-IR (NIR) $J H K_{s}$ data in the database. 
Table 1

Overview of Studies and Data Included

\begin{tabular}{|c|c|c|c|}
\hline Data Set & $\begin{array}{l}\text { Year Published } \\
\text { (or Obtained) }\end{array}$ & Band(s) & Notes \\
\hline ASR & 1994 & $J H K$ & Many coordinate issues; see text \\
\hline LAL & 1996 & JHK & Many coordinate issues; see text \\
\hline Preibisch & 1997 & $\mathrm{X}$-ray $(R O S A T)$ & Two coordinate issues; source 2 may be spurious \\
\hline VLA & 1999 & $3.6,6 \mathrm{~cm}$ & Hard to find short-wavelength counterparts \\
\hline 2MASS & $(2000)$ & $J H K_{s}$ & $\begin{array}{l}\text { Data obtained in } 2000 \text { as part of } 2 \text { MASS all-sky survey and subsequently the } \\
\text { 2MASS } 6 \times \text { survey }\end{array}$ \\
\hline Getman (et al.) & 2002 & $\mathrm{X}$-ray (Chandra/ACIS) & Two inconsistencies \\
\hline Preibisch & 2003 & $\mathrm{X}$-ray $(X M M)$ & Most sources match Chandra sources \\
\hline Rebull (et al.) & 2003 & MIR & Primary information included from this work is source multiplicities \\
\hline Aspin & 2003 & NIR & Spectroscopy; spectral types included \\
\hline MBO & 2004 & $J H K$ & Duplicate MBO number \\
\hline $\mathrm{c} 2 \mathrm{~d}$ & 2006, 2007 & Spitzer: 3.6 to $70 \mu \mathrm{m}$ & Included limits and $70 \mu \mathrm{m}$ sources \\
\hline Hatchell (et al.) & 2007 & $450,850 \mu \mathrm{m}$ & Cross IDs retained \\
\hline Greissl (et al.) & 2007 & $J H$ & NICMOS brown dwarf search \\
\hline Gutermuth (et al.) & 2008 & Spitzer: 3.6 to $24 \mu \mathrm{m}$ & Only YSO candidates reported \\
\hline Oasa (et al.) & 2008 & $J H K_{s}$ & Brown dwarf search \\
\hline Scholz (et al.) & $2009,2012 \mathrm{ab}$ & $i^{\prime} z^{\prime} J K$ & SONYC; brown dwarf search \\
\hline Winston (et al.) & 2009,2010 & $\begin{array}{l}\text { Spitzer/IRAC } \\
\quad+\text { Chandra/ACIS }\end{array}$ & Some potentially confusing source numbers in literature; fixed here \\
\hline Itoh (et al.) & 2010 & optical & Spectroscopy of brown dwarf candidates \\
\hline WISE & $(2010)$ & $3.5,4.6,12,22 \mu \mathrm{m}$ & $\begin{array}{l}\text { Data obtained in } 2010 \text { as part of All-Sky Survey; AllWISE reduction used, but only } \\
\text { in certain very specific cases (not broadly nor blindly used). }\end{array}$ \\
\hline Arnold (et al.) & 2012 & MIR & Spectroscopy with Spitzer IRS \\
\hline 3XMM-DR4 & 2013 & $\mathrm{X}$-ray $(X M M)$ & All-sky catalog from $X M M$ \\
\hline Foster (et al.) & 2015 & NIR & Spectroscopy with APOGEE \\
\hline Sadavoy (et al.) & 2014 & FIR & Class 0 Herschel cross-identifications \\
\hline YSOVAR & 2014 & $3.6,4.5 \mu \mathrm{m}$ & $\begin{array}{l}\text { Means from YSOVAR monitoring included; variables not yet recognized as YSOs } \\
\text { are included here }\end{array}$ \\
\hline
\end{tabular}

\subsection{Cryogenic-era Spitzer Archival Data}

Early in the Spitzer mission, NGC 1333 was observed by both the guaranteed time observations (GTO) and the original Cores-to-Disks (c2d) Legacy program (Evans et al. 2003, 2009). For both the Infrared Array Camera (IRAC; Fazio et al. 2004) and Multiband Imaging Photometer for Spitzer (MIPS; Rieke et al. 2004) data, the observations were obtained at multiple epochs separated by at least a few hours to allow asteroids to move and thus be identified as asteroids (as opposed to embedded objects in NGC 1333). The IRAC observations are at $3.6,4.5,5.8$, and $8 \mu \mathrm{m}$; the MIPS observations are at 24,70 , and $160 \mu \mathrm{m}$, but the $160 \mu \mathrm{m}$ data are very difficult to interpret in this region and are not included here. The first IRAC observation was part of GTO program 6, obtained on 2004 February 10; a second group of IRAC observations was part of c2d, program 178, on 2004 September 08. The first MIPS observations were part of GTO program 58, obtained on 2004 February 03; an additional three epochs were part of c2d program 178, obtained on 2004 September 19 and 20.

As discussed in R14, the cryogenic data were combined and reduced identically to the YSOVAR monitoring data, except using cryogenic calibrations and combining the two observations into a single effective epoch (rather than maintaining separate measurements for each epoch). The apertures we used were 2". 4. The data were band merged across Spitzer bands by position, and then to the $2 \mathrm{MASS}$ catalog, within a search radius of $1^{\prime \prime}$.
Gutermuth et al. (2008a, 2009, 2010) present methodology for identifying YSOs from the cryogenic catalog. The details of the selection process appear in those papers, but in summary, multiple cuts in multiple color-color and color-magnitude diagrams are used to identify YSO candidates, as distinct from, e.g., extragalactic and nebular contamination. This color selection process was run anew on the re-reduced data.

Spitzer data are also available from the c2d program final data delivery. The data used for the final delivery are typically the same BCDs as were used in the cryogenic data that we rereduced above. As such, then, they are not independent measurements, and these data were only used to supplement the cryogenic-era catalog if a band was missing. There is more information on what I extracted from the c2d catalogs below.

\subsection{Chandra ACIS}

Chandra X-ray Observatory Advanced CCD Imaging Spectrometer for wide-field imaging (ACIS-I) observations of NGC 1333 were first reported in Getman et al. (2002) and then Winston et al. (2009, 2010). There are three pointings in NGC 1333, with obs IDs 642, 6436, and 6437, with a total exposure time of $119.3 \mathrm{ks}$.

As was described in R14, we re-reduced the Chandra data in a self-consistent way across most of the YSOVAR clusters. Source detection was performed using CIAO (Chandra Interactive Analysis of Observations; Fruscione et al. 2006). Sources, even faint ones, were retained if they had a counterpart in the cryogenic IRAC catalog. Sources from 
Chandra were matched to the rest of the catalog with a position-dependent search radius; see R14.

Cross-IDs from these X-ray papers are included; see below. However, the X-ray flux measurement data are included in their entirety in L. M. Rebull et al. (2015, in preparation), the paper on the NGC 1333 YSOVAR data, and do not play a role in the SEDs, so they are not explicitly included here. As R14 describes, we identify some candidate cluster members by looking for objects with star-like SEDs and an X-ray detection. There are only two new X-ray candidate cluster members that are introduced as part of this process (J032913.47+312440.7 and J032837.85+312525.3); all of the other so-identified members were identified in the literature as (candidate) members already.

\subsection{WISE}

The Wide-field Infrared Survey Explorer (WISE; Wright et al. 2010) surveyed the whole sky at 3.5, 4.6, 12, and $22 \mu \mathrm{m}$; all of the available WISE data taken between 2010 January and 2011 February were incorporated into the AllWISE catalog. WISE has lower spatial resolution than Spitzer and is on average less sensitive. I do not generally include the AllWISE data, since NGC 1333 is often a complicated region with high surface brightness, and because we have extensive higher spatial resolution Spitzer data. However, WISE provides a band at $12 \mu \mathrm{m}$ that is not available from Spitzer. I have incorporated WISE data for certain individual sources, where viable photometry is available from WISE and the photometry from Spitzer is incomplete or results in an unusual SED shape. (For example, in some cases, the [24] point seemed unphysical in the context of the SED, but the [22] point is well matched to it.) Comparison of the WISE images with the Spitzer images was also useful in certain circumstances, such as for investigating the influence of image artifacts-artifacts change between Spitzer and WISE, but sources on the sky should not.

I use the AllWISE data release in nearly all cases, but I now note an exception. In one very crowded region (sources that are components of IRAS 7), the WISE All-Sky catalog does a better job of separating the sources than the AllWISE catalog. AllWISE seems to have inferred that there was significant proper motion of one of the sources in the clump, and by inspection of the images, this is not correct. Thus, WISE flux densities for the catalog sources J032910.70+311820.9 and J032911.24+311831.8 are taken from the All-Sky catalog, not the AllWISE catalog.

\section{LITERATURE DATA AND SOURCE RECONCILIATION}

Many studies have been made specifically of NGC 1333-it is one of the most well-studied star-forming regions, with $>200$ refereed publications in ADS. It is difficult to include data from every single paper, especially since so many papers focus on just one or a few objects in the region, or on just extended objects (e.g., Raga et al. 2013). I endeavored to include in the catalog the most recent catalogs of point sources and/or those that had the largest repositories of data, and/or those that were the origin of some source names still in common use today. The majority of the information actually included in the database from the earliest studies is the cross-identifications (cross-IDs) with the literature; additional broadband photometry was included where possible, and not superceded by subsequent reprocessing of the data. In the process of
Table 2

Contents of Cross-ID Catalog

\begin{tabular}{|c|c|c|}
\hline Num & Label & Explanations \\
\hline 1 & cat & $\begin{array}{l}\text { Catalog name (HHMMSS.ss+DDMMSS.s; } \\
\text { J2000); should be preceeded by "R15- } \\
\text { NGC } 1333 J \text { " }\end{array}$ \\
\hline 2 & ASR & ASR number \\
\hline 3 & ASR-YSO? & $\begin{array}{l}\text { Did ASR tag it as a YSO? } 1=\text { yes, } 0=\text { no, } \\
-9 \text { no information }\end{array}$ \\
\hline 4 & LAL & LAL number \\
\hline 5 & LAL-YSO? & $\begin{array}{l}\text { Did LAL tag it as a YSO? } 1=\text { yes, } 0=\text { no, } \\
-9 \text { no information }\end{array}$ \\
\hline 6 & VLA & VLA number \\
\hline 7 & VLA-YSO? & $\begin{array}{l}\text { Did Rodriguez et al. tag it as a YSO? } 1=\text { yes, } \\
0=\text { no, }-9 \text { no information }\end{array}$ \\
\hline 8 & Preibisch & Preibish number \\
\hline 9 & 2MASS & 2MASS name \\
\hline 10 & Getman & Getman number \\
\hline 11 & Getman-var & $\begin{array}{l}\text { Did Getman tag it as variable } ? 1=\text { yes, } \\
0=\text { no, }-9 \text { no information }\end{array}$ \\
\hline 12 & MBO & MBO number \\
\hline 13 & $\mathrm{c} 2 \mathrm{~d}$ & c2d name \\
\hline 14 & c2d-YSO? & $\begin{array}{l}\text { Did c2d tag it as a YSO? } 1=\text { yes, } 0=\text { no, }-9 \\
\text { no information }\end{array}$ \\
\hline 15 & Greissl & Greissl et al. (2007) number \\
\hline 16 & Hatchell & Number from Hatchell et al. (2007) \\
\hline 17 & Oasa & Number from Oasa et al. (2008) number \\
\hline 18 & Oasa-YSO? & $\begin{array}{l}\text { Did Oasa et al. tag it as a member? } 1=\text { yes, } \\
0=\text { no, }-9 \text { no information }\end{array}$ \\
\hline 19 & G08 & Number from Gutermuth et al. (2008) \\
\hline 20 & G08-YSO? & $\begin{array}{l}\text { Did Gutermuth et al. tag it as a member? } \\
1=\text { yes, } 0=\text { no, }-9 \text { no information }\end{array}$ \\
\hline 21 & S09 & Number from Scholz et al. (2009) \\
\hline 22 & Winston & Number from Winston et al. (2010) \\
\hline 23 & S12 & Number from Scholz et al. (2012a) \\
\hline 24 & S12-YSO? & $\begin{array}{l}\text { Did Scholz et al. tag it as a member? } 1=\text { yes, } \\
0=\text { no, }-9 \text { no information }\end{array}$ \\
\hline 25 & A12 & Number from Arnold et al. (2012) \\
\hline 26 & A12-YSO? & $\begin{array}{l}\text { Did Arnold et al. tag it as a YSO? } 1=\text { yes, } \\
0=\text { no, }-9 \text { no information }\end{array}$ \\
\hline 27 & Foster & $\begin{array}{l}\text { Number from Foster et al. (2015, in } \\
\text { preparation) }\end{array}$ \\
\hline 28 & YSOVAR & $\begin{array}{l}\text { YSOVAR name from L. M. Rebull } \\
\text { et al. (2015) }\end{array}$ \\
\hline 29 & $\begin{array}{l}\text { YSOVAR- } \\
\text { YSO? }\end{array}$ & $\begin{array}{l}\text { Did Rebull et al. tag it as a YSO? } 1=\text { yes, } \\
0=\text { no, }-9 \text { no information }\end{array}$ \\
\hline 30 & Other & Any other names or cautions for this object \\
\hline
\end{tabular}

(This table is available in its entirety in machine-readable and Virtual Observatory (VO) forms.)

assembling the literature catalog of sources, I reconciled many ambiguities and inconsistencies in the literature. I provide below descriptions of the more complicated issues. All of the reconciled cross-identifications in NGC 1333 are included in Table 2, including an indication of whether or not the corresponding survey identified the object as a YSO.

All of the aggregate $J$-[24] single-epoch photometry appears in Table 3 . There are nearly 7000 objects in the catalog, about 300 of which are identified in the literature as YSO candidates.

IAU standards recommend not renaming previously identified sources, but as one of the purposes of the present catalog is to sort out inconsistencies and inaccuracies in existing catalogs, assigning a new, coordinate-based name seems appropriate. The coordinate-based names presented here (in Tables 2 and 3) should be preceeded by "R15-NGC 1333." 
Table 3

Contents of Single-epoch 1-70 $\mu \mathrm{m}$ Catalog

\begin{tabular}{|c|c|c|c|}
\hline Num & Units & Label & Explanations \\
\hline 1 & $\cdots$ & cat & Catalog name (HHMMSS.ss+DDMMSS.s; J2000) \\
\hline 2 & $\operatorname{deg}$ & RA & R.A., J2000, decimal degrees \\
\hline 3 & $\operatorname{deg}$ & Dec & Decl., J2000, decimal degrees \\
\hline 5 & mag & $\bar{J}$ & $J$-band magnitude \\
\hline 6 & mag & $\mathrm{e} \_J$ & Uncertainty in $J$ \\
\hline 7 & $\cdots$ & $1 \_H$ & Limit flag on $H$ \\
\hline 10 & $\ldots$ & $1 \_K_{s}$ & Limit flag on $K_{s}$ \\
\hline 11 & mag & $\overline{K_{s}}$ & $K_{s}$-band magnitude \\
\hline 12 & mag & $\mathrm{e} \_K_{s}$ & Uncertainty in $K_{s}$ \\
\hline 13 & $\cdots$ & $1 \_[3.6]$ & Limit flag on [3.6] \\
\hline 14 & mag & {$[3.6]$} & Spitzer/IRAC $3.6 \mu \mathrm{m}$ band magnitude \\
\hline 15 & mag & e_[3.6] & Uncertainty in $[3.6]$ \\
\hline 20 & mag & {$[5.8]$} & Spitzer/IRAC $5.8 \mu \mathrm{m}$ band magnitude \\
\hline 21 & mag & e_[5.8] & Uncertainty in $[5.8]$ \\
\hline 22 & $\cdots$ & $1 \_[8.0]$ & Limit flag on $[8.0]$ \\
\hline 23 & mag & {$[8.0]$} & Spitzer/IRAC $8.0 \mu \mathrm{m}$ band magnitude \\
\hline 24 & mag & e_[8.0] & Uncertainty in $[8]$ \\
\hline 25 & $\ldots$ & $1 \_[24]$ & Limit flag on [24] \\
\hline 26 & mag & {$[24]$} & Spitzer/MIPS $24 \mu \mathrm{m}$ band magnitude \\
\hline 27 & mag & e_[24] & Uncertainty in $[24]$ \\
\hline 28 & $\cdots$ & $1 \_[70]$ & Limit flag on $[70]$ \\
\hline 29 & mag & {$[70]$} & Spitzer/MIPS $70 \mu \mathrm{m}$ band magnitude \\
\hline 30 & mag & e_[70] & Uncertainty in $[70]$ \\
\hline 31 & $\ldots$ & SpType & Spectral type \\
\hline 40 & $\cdots$ & H07 & SED class from Hatchell et al. (2007) \\
\hline 41 & $\cdots$ & S14 & SED class from Sadavoy et al. (2014) \\
\hline
\end{tabular}

(This table is available in its entirety in machine-readable and Virtual Observatory (VO) forms.)

Figure 1 gives a rough indication of the various larger surveys included here. The footprints from Chandra and from the YSOVAR monitoring are shown close to their actual coverage. No attempt is made to capture complex polygons of coverage for the other surveys, but just squares encompassing all sources are shown; in other words, there are no data from a given survey outside of its square, but there may be incomplete spatial coverage inside of it.

\subsection{On Coordinate Accuracies}

Integral to the process of source matching across catalogs is an understanding of the systematic and statistical errors present in the positions of the objects in the catalogs. 2MASS provides a very high quality coordinate system, $<0$ ". 1 with respect to the International Celestial Reference System (ICRS) reference frame for bright sources, over the whole sky. WISE and Spitzer coordinates are fundamentally tied to this 2MASS coordinate system. WISE positional uncertainties are typically $<0$ ". 2 , often much less. Spitzer/IRAC positional uncertainties are comparable at $<0$ "' 2 .

I present the prior studies I integrated in this section in roughly chronological order. However, in practice, I iteratively (and very often manually) compared each of the sources in the older catalogs to the 2MASS catalog and images, adjusting or correcting coordinates as needed, before merging all the catalogs together. In many cases, it was a simple shift within $\lesssim 5^{\prime \prime}$ from a place on the sky without a 2MASS source to the location of a relatively bright 2MASS source. I note that the Infrared Science Archive (IRSA) tool FinderChart ${ }^{1}$ was extremely helpful for this process. FinderChart uses data from WISE, 2MASS, and the DSS, which is a digitization of the

\footnotetext{
1 http://irsa.ipac.caltech.edu/applications/finderchart/
} 


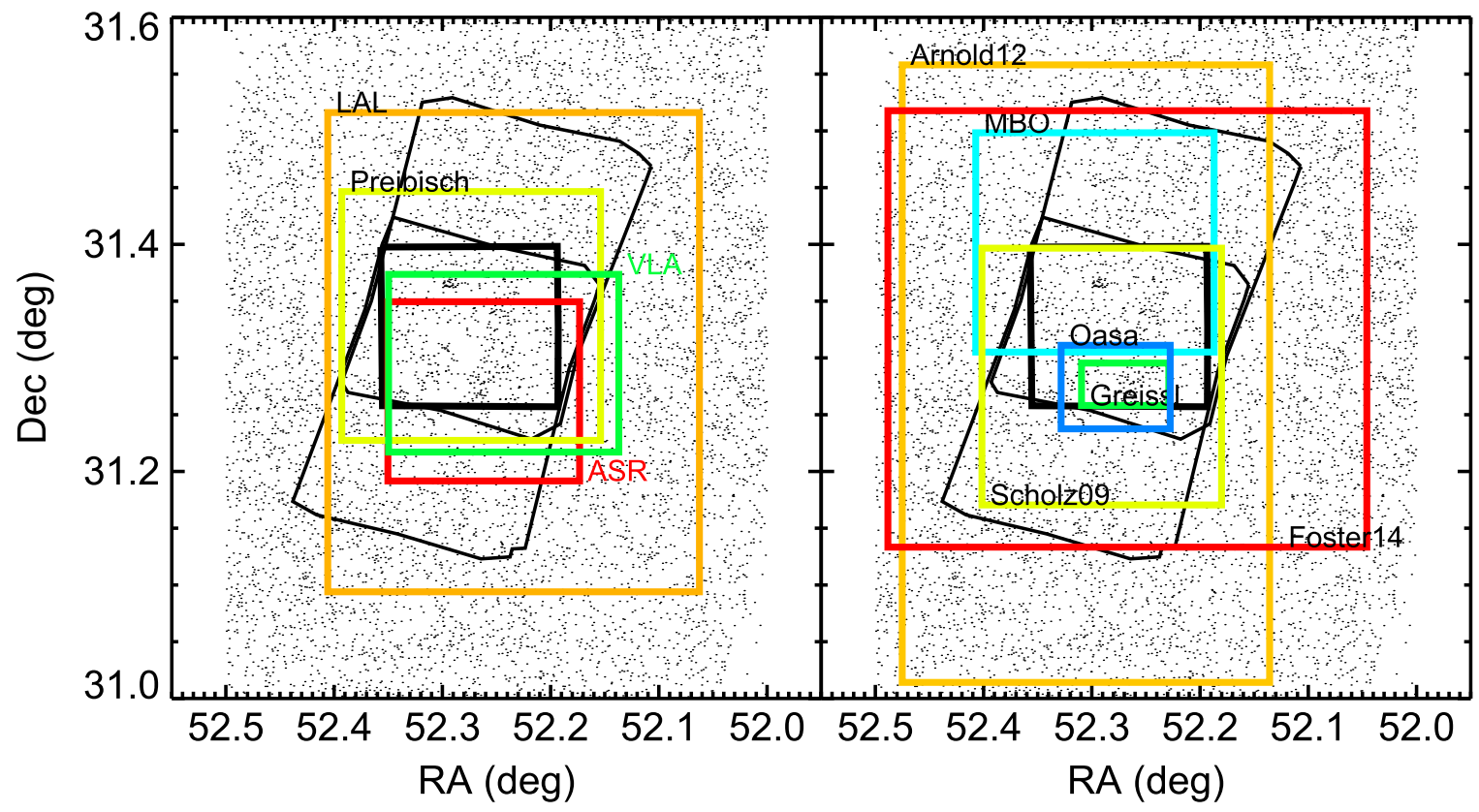

Figure 1. Approximate spatial coverage of the various catalogs included in this survey, distributed over two plots simply for clarity. Each small black dot corresponds to an object in the final catalog assembled here. The black polygons in both plots are the coverage of the 3.6 and $4.5 \mu$ m YSOVAR monitoring regions, and the thick black square in both plots is the Chandra coverage. Colored squares correspond roughly to most of the other surveys included here, as indicated in the corners of the squares. For these colored squares, note that what is shown is the $\min / \max$ of the R.A./decl.; no attempt is made at polygon representation of the other survey regions, because this figure is simply meant to give a rough indication of the regions of the cluster observed in these respective surveys, e.g., a relatively small region has been probed with NICMOS (the green Greissl et al. square).

photographic sky survey plates from the Palomar (Palomar Observatory Sky Survey) and UK Schmidt telescopes. FinderChart provides a thumbnail image of the sky at multiple wavelengths, making comparisons relatively straightforward.

For any catalog, the coordinate accuracy depends on there being sufficient numbers of point sources in the field of view (or mosaic) in order to anchor the coordinate system. This has two ramifications for the catalogs considered in this section. First, for some early NIR observations, arrays were very small (for example, Aspin et al. 1994 had a single pointing field of view of just under an arcminute on a side), resulting in relatively few point sources per pointing, making astrometry very hard to do accurately. Moreover, then as now, astrometry was bootstrapped to prior observations, but at that time, this bootstrapping had to occur without the reliable all-sky anchor provided by 2MASS. Second, for long-wavelength observations such as Spitzer/MIPS and Herschel maps, there are many fewer point sources that can be linked to the 2MASS coordinate system, so the positional uncertainty can be worse, and sometimes astrometry relative to one or a few point sources is the best available. It can be difficult to make a clear correspondence between the shorter-wavelength and longerwavelength sources, not just because of the coordinates, but also because the emission may not be coming from the same location in/near the object. I have attempted to make these matches here, being mindful of the fact that these longwavelength sources are often in regions that are extremely complex, with high surface brightness (complicating both photometry and astrometry). Moreover, the source of the longwavelength emission may just be by chance aligned with an emitter of short-wavelength emission. I believe what I have done is correct, but I have provided descriptions below of what I have done in the event that subsequent investigators disagree.
Another source of positional uncertainty is the space motions of these objects. Over the time baselines considered here, could the sources in NGC 1333 be moving significantly? Karchenko et al. (2013) report that for NGC 1333 the average $\mu_{\text {R.A. }}, \mu_{\text {decl. }}=5.51,-10.28$ mas yr $^{-1}$, so over $50 \mathrm{yr}$ (longer than considered here), a typical object could move $\sim 0^{\prime \prime} 5$. However, the spatial resolution for both 2MASS and IRAC is $\sim 1^{\prime \prime}$, and these catalogs are the input catalogs that establish the bulk of the master NGC 1333 catalog. I search for counterparts between catalogs with a typical tolerance of $\sim 1^{\prime \prime}$. Therefore, most sources will not have enough proper motion over the baselines considered here to affect the catalog merging. Assessing the motion of these objects based on these data is theoretically possible, but beyond the scope of this work.

\subsection{ASR Catalog}

Aspin et al. (1994; ASR) was one of the first wide-field NIR surveys of NGC 1333 reported in the literature. They published a full $J H K$ catalog (i.e., not just the objects they thought were cluster members) for 134 objects to $K=16.2$. I incorporated their full catalog into my database, keeping track of the objects tagged by ASR as likely YSOs. Numbers from this catalog are preceeded by "ASR." JHK measurements from this catalog were not retained because there were 2MASS measurements available. Moreover, the photometry on average is well matched, typically within $\sim 0.2 \mathrm{mag}$ (often less); while most of the stars in the catalog are unlikely to be members, young stars are expected to be intrinsically variable at levels greater than a few tenths of a magnitude.

Since this is the first (and oldest) literature catalog I consider here, I report some additional information on it as representative of the difficulties inherent in this process and my approach to it. While an individual field of view was just under an 


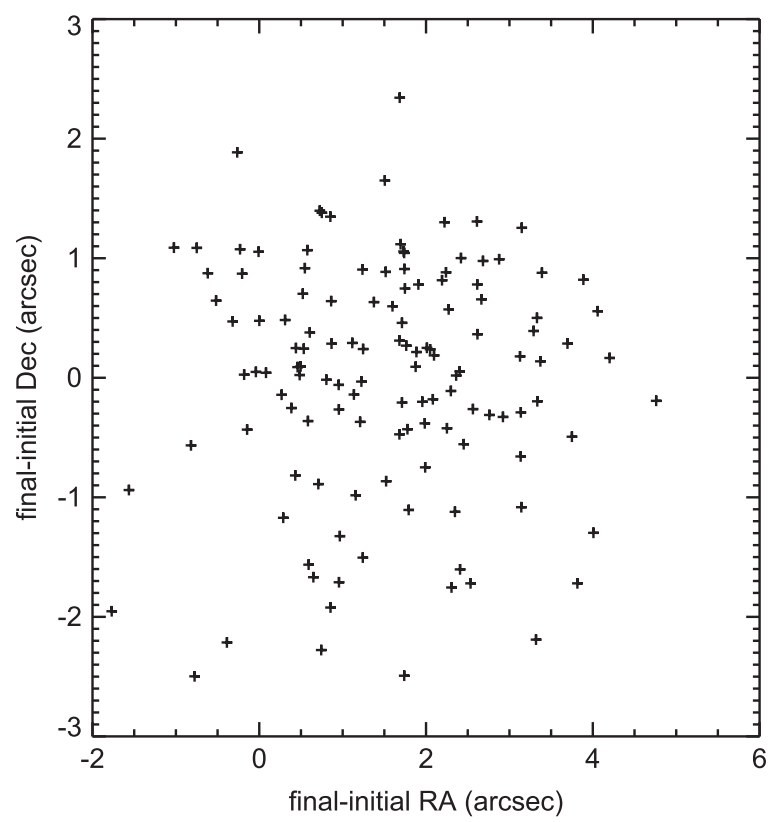

Figure 2. Comparison of offsets in R.A. and decl. (both in arcseconds) between the final, adopted position (matching 2MASS) and the intitial position precessed from the B1950 coordinates provided in ASR. The offests are symmetric in declination and reveal a systematic offset in R.A. The median net offset is $\sim 1$ !"7.

arcminute on a side, ASR mapped $\sim 10^{\prime} \times 10^{\prime}$. They established astrometry by comparison to Herbig \& Jones (1983) for 30 objects, estimating uncertainties of $\sim 1^{\prime \prime}$ as a result. The original ASR catalog was reported in B1950 coordinates, which I precessed and overlaid on the images and compared to the 2MASS and IRAC catalogs. ASR was a relatively shallow $K$ band observation, comparable to 2MASS classic (as opposed to 2MASS 6x). These sources (particularly the NGC 1333 members) are intrinsically variable. However, while I expected that variability at $K$ could make the object appear or disappear out of a relatively shallow survey, I still expected it to show up at the 2MASS $6 \times$ depth or at least in the IRAC maps, not necessarily gone entirely. The IRAC observations reach at the very least the same sources as 2MASS, for most SEDs; even relatively shallow IRAC observations commonly reach sources fainter than are detected by 2MASS. For nearly $90 \%$ of the 134 ASR sources, I could find counterparts within $5^{\prime \prime}$ in the 2MASS catalog, with a median positional offset of $\sim 1$ !' 7 , and larger offsets in R.A. than in decl.; see Figure 2. For most of the sources, particularly for those with the larger positional offsets, images from 2MASS were compared to the positions provided in the original ASR to ensure that the match was appropriate. In most cases, the match was readily apparent.

Unlike most of the rest of the cluster, the region containing the trio of ASR 31/32/33 is quite confusing, as can be seen in Figure 3. I now discuss this region in detail.

ASR 33 is the easiest of this triplet and is probably the same as MBO 171 (which is the same cross-identification provided by Wilking et al. 2004). It is catalog source R15-NGC 1333 J032911.51+311828.6.

VLA 27 has been tied (including by myself in Rebull et al. 2003) to one of the ASR 31/32/33 sources. But note that there is an IRAC source coincident with the position reported for VLA 27 , offset (by $\sim 4 . .5$, a significant amount by IRAC standards) from ASR 31/32/33. And, note that there is no
2MASS source (in the shallower or deeper 2MASS data). The original ASR survey could not have been enough deeper than 2MASS $6 x$ to detect a source not recovered by either of the 2MASS surveys; intrinsic variations of this level in this source are possible but unlikely. I have tied VLA 27 to the IRAC source (R15-NGC 1333 J032700.47+313725.9), but not the ASR sources. The VLA positions should be very good, so leaving this source affiliated with the IRAC source with which it is coincident seems appropriate.

There is a rough arc of $2 \mathrm{MASS}$ sources with the same rough concavity as the arc of ASR 31/32/33 sources. But there are five total 2MASS sources, and only three ASR sources. Could the northern three 2MASS sources be the three ASR sources? Wilking et al. (2004), a.k.a. the MBO source numbers below, linked ASR 31 to MBO 161. Given the catalog sources overlaid in Figure 3, MBO 161 is not the same as ASR 31.

The original ASR astrometry has some uncertainties, but not as bad as would be required to enforce the match of the two arcs of sources, especially over this small region. I obtained the original data from ASR (C. Aspin 2015, private communication), which had been updated with more recent astrometry; see Figure 3. There are two sources in the left corner of the ASR data bright enough to be in 2MASS classic. Note that the originally reported MBO, IRAC, and 2MASS source positions are a little to the south of the northern source and a little to the north of the southern source, which should be representative of the net positional errors in this immediate region. The original ASR coordinates in this region also reflect this tiny distortion in the north-south direction, less so in the east-west direction. The local astrometry is probably correct (unless there is a tile boundary here, which does not seem to be the case in the ASR image; see also LAL coordinate matching below). So the differential astrometry should be high quality among the local ASR sources. There is nothing approaching the magnitude of the distortions that would be required to map ASR 31 onto MBO 161 and ASR 32 onto the 2MASS source that would be in between the two other ostensible ASR matches.

I conclude that:

1. ASR 31 should be matched to catalog source J032911.67 +311832.3 (and c2d source J032911.6+311832).

2. ASR 32 is likely spurious and cannot be tied to any source detected in these other surveys to date, so I have removed it from the catalog. It may be that it should be matched to VLA 27 (others have made this match in the literature), but I have not enforced this match here.

3. ASR 33 should be matched to MBO 171 and catalog source J032911.51+311828.6 (and c2d source J032911.5 +311828 ).

4. VLA 27 should match to catalog source J032911.24 +311831.8 (and c2d source J032911.3+311831).

5. ASR 31 should not be matched to MBO 161 (which is different than what is found in the literature). MBO 161 is matched to the catalog source J032912.13+311832.1 (and c2d source $\mathrm{J} 032912.1+311832$ ).

Another more minor ASR issue pertains to ASR 50, which is clearly in the original ASR image and can be seen in 2MASS $6 \times$, but is on a diffraction spike and resembles an artifact enough that it was probably dropped by the 2MASS pipeline as a result. There is an IRAC source within 2."5 of the Aspin source $(\mathrm{J} 032855.08+311416.4)$ that is most likely the match. 

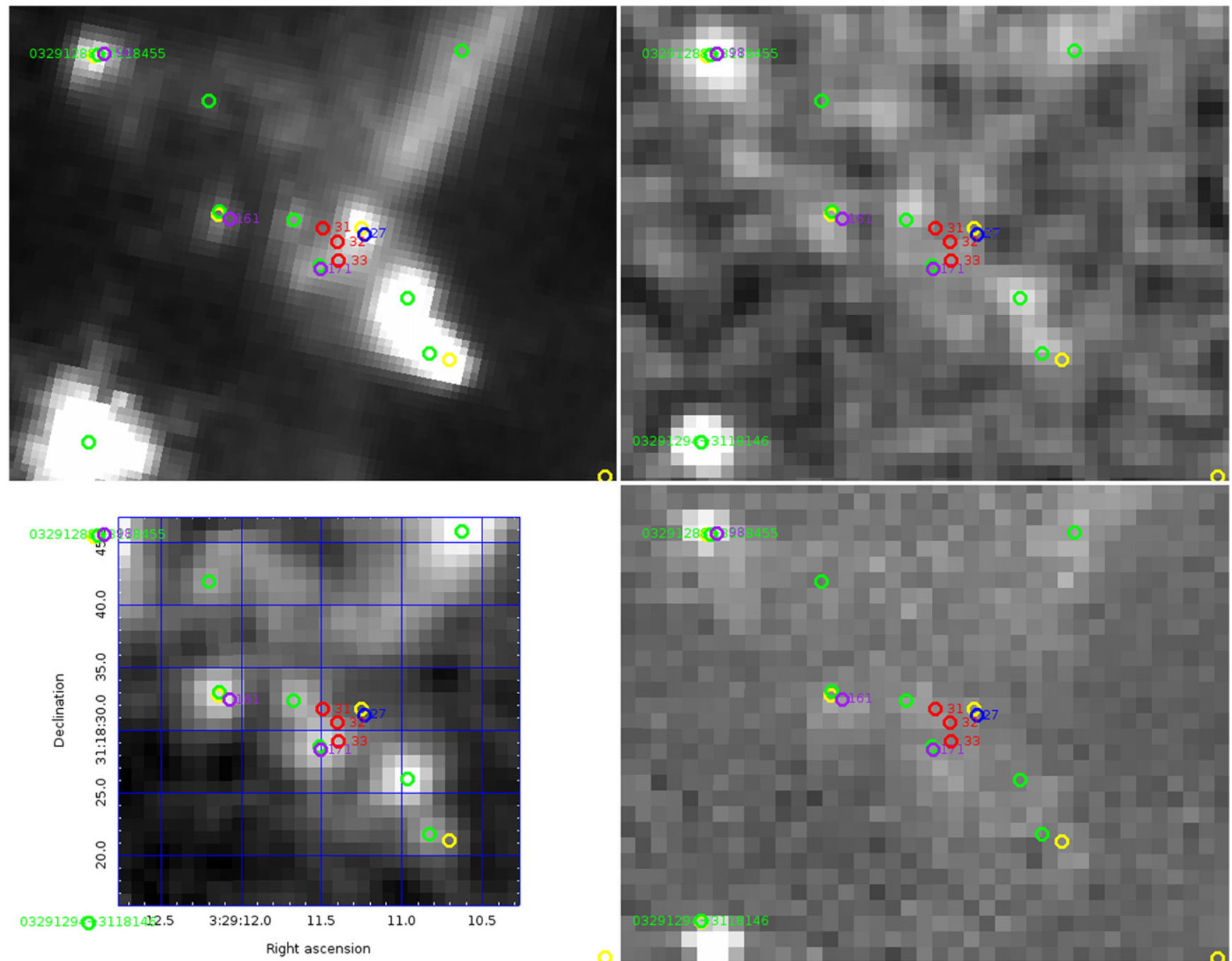

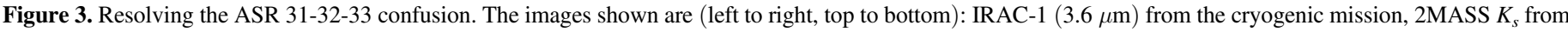

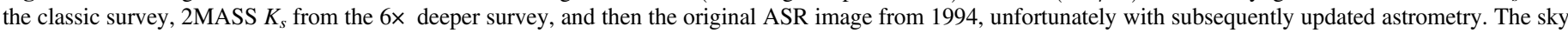

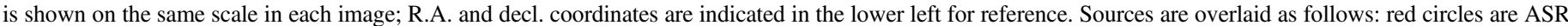

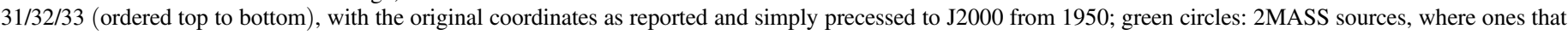

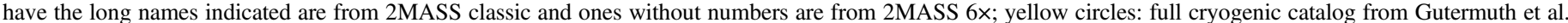

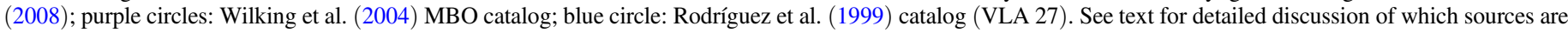
identified with each other in the ASR 31-32-33 region.

There are several remaining ASR sources for which 2MASSclassic counterparts cannot easily be found. ASR $5=\mathrm{J} 032904.53+311554.6$, oddly, can be seen clearly in the DSS images, but by 2MASS $J H K_{s}$ is extremely faint and is lost in the glare of a nearby very bright source by Spitzer and WISE bands. I have retained it with the original ASR coordinates, since it can be clearly identified in the DSS and is well matched to that position. ASR 20,21, and 22 are all in a region that becomes bright with extended emission at Spitzer bands. ASR 20 does not have a clear match in 2MASS, but ASR 21 is faintly seen in the 2MASS $K_{s}$ images and may be extended. There are two sources from the $6 \times$ catalog (both of which may be part of the extended emission); ASR 21 is matched to the closer one. At $3.6 \mu \mathrm{m}$, ASR 22 is close to a blob of extended emission that may have concentrated knots within it; I have tied it to 2MASS J03290842+3115284 as the brightest source in the blob. ASR 71 is entirely within another region that, at $3.6 \mu \mathrm{m}$, is a blob of extended emission that may have concentrated knots within it. The brightest portion of the blob is already identified with ASR 49. It is unclear to what object at 2MASS bands ASR 71 should be matched, so I have retained it with the original ASR coordinates. ASR 119 is almost exactly in between two 2MASS sources that are very far away, one $\sim 15^{\prime \prime}$ north and one $\sim 18^{\prime \prime}$ south. Lacking a compelling reason to look this far away for a counterpart and make the association with one or the other, I have left ASR 119 alone at its original location despite having no counterpart. ASR 133 is similiarly more or less in between two sources $\sim 15^{\prime \prime}$ north and south of this position; similarly, I have left it alone. ASR 134 does not have a 2MASS-classic counterpart, but can be seen faintly on the $K_{s}$ images, and it is bright by IRAC bands. ASR 50,75, 94, and 98 all have IRAC matches within 5", even if counterparts are not apparent in the 2MASS-classic images.

\subsection{LAL Catalog}

Lada et al. (1996; LAL) also imaged NGC 1333 in JHK, over a region 4 times larger in area than ASR. Their entire 


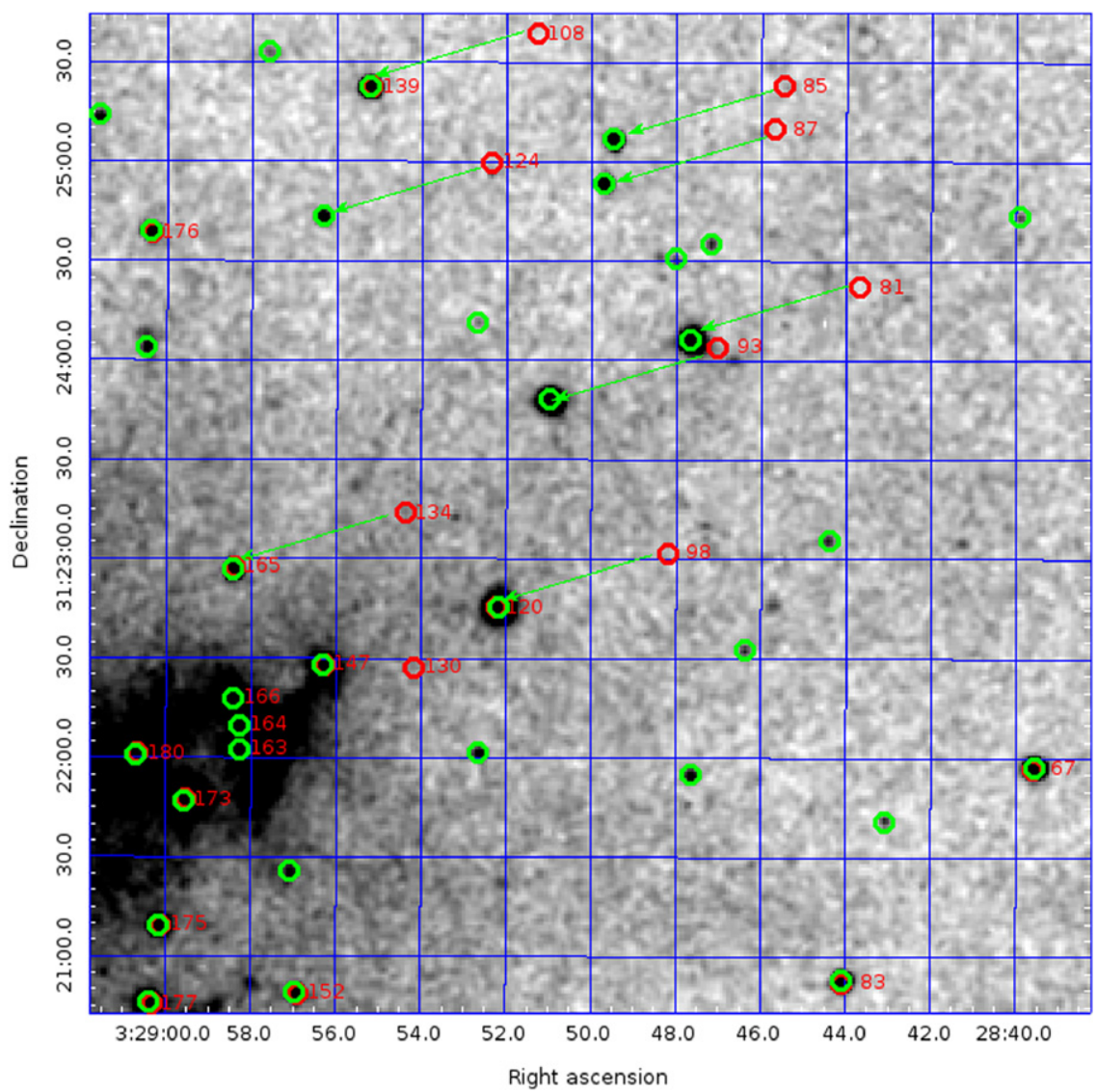

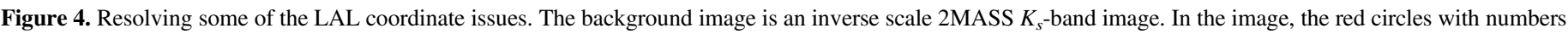

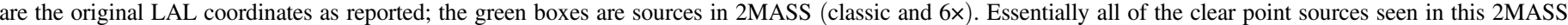

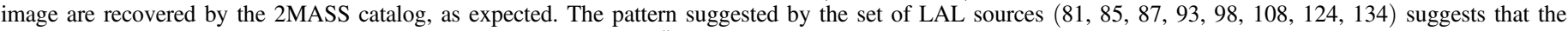

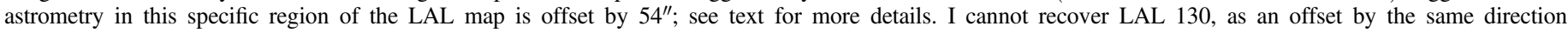
and magnitude does not land on a 2 MASS source.

catalog was not included in the original article, but was obtained via private communication from C. Lada. Objects LAL thought were young were also indicated in this catalog. Numbers from this catalog are preceeded by "LAL." JHK measurements from this catalog were not retained because 2MASS measurements were available for all of the retained LAL sources. As for ASR above, the ensemble of $J H K$ photometry is reasonably well matched to 2MASS (better than $\sim 0.2 \mathrm{mag}$ ), though there can be excursions for individual sources.

The observations presented in LAL were assembled from many smaller pointings in the NIR, though unlike ASR, a single pointing field of view for LAL was relatively large, and at $\sim 5^{\prime}$, comparable to a single IRAC field of view. LAL report that their astrometry is based on five stars from the Hubble Space Telescope (HST) Guide Stars Catalog, and that their estimated positional uncertainty is $0 . \prime 5 \pm 0$ !. 2 . However, based on my comparison to 2MASS, I strongly suspect that there are several astrometric problems. In most cases, the sources are recoverable; for $86 \%$ of the sources, I can find a counterpart in 2MASS within $3^{\prime \prime}$. However, in some cases, I could not find a counterpart. In one region, I noticed a pattern offset, which I now describe.

As seen in Figure 4, I strongly suspect, based on relative positions, that several sources in the LAL catalog should all move $54^{\prime \prime}$ to the southeast. These sources all appear, perhaps, to be in the corner of a component tile of the LAL final mosaic, and evidently the astrometry in this region (tile corner?) was not well constrained. The consistency in the pattern of sources on the sky is compelling evidence that the sources should be shifted; a direct comparison of the $J H K_{s}$ values on a source-bysource basis often supports this assertion.

The repercussions are that:

1. LAL 81 moves from having no match to $2 \mathrm{MASS}$ J03284764+3124061, which is nominally close to the original LAL 93 position. There is a faint source that appears only at $3.6 \mu \mathrm{m}$ under this originally reported location of LAL 81, but the SED for that object is different from the LAL-reported $J H K$ by many orders of magnitude, which was what originally led me to suspect that this was not the correct match. The LALreported $J H K$ matches the 2MASS photometry reasonably well, being offset by $0.16,0.12$, and $0.02 \mathrm{mag}$ in $J$, $H$, and $K$, respectively.

2. LAL 85 moves from having no match to $2 \mathrm{MASS}$ J03284947+3125066. The photometry matches between these sources to $0.14 \mathrm{mag}$ in $J, 0.08 \mathrm{mag}$ in $H$, and $0.01 \mathrm{mag}$ in $K$.

3. LAL 87 moves from having no match to 2 MASS J03284971+3124534. The magnitudes match to 0.24 , 0.10 , and 0.06 mag in $J, H$, and $K$.

4. LAL 93 moves from 2MASS J03284764+3124061 (where it is offset from a bright star, and to which it has sometimes been tied in the literature) to 2MASS 
J03285097+3123479. The photometry matches the latter source, 2MASS J03285097+3123479, better $(0.16,0.04$, 0.01 in $J H K)$ than the former source, 2MASS J03284764 $+3124061(0.17,0.22,0.41$ in $J H K)$.

5. There is nothing at the nominal location of LAL 98. If I apply the offset in the same direction as the other sources above, then LAL 98 is identical to LAL 120, which is already matched to 2MASS J03285216+3122453. However, the LAL reported magnitudes for 98 and 120 differ by $\sim 1$ mag at each band. On the other hand, 2MASS $\mathrm{J} 03285216+3122453$ has measured $J H K_{s}$ within $0.19 \mathrm{mag}$ at all bands to LAL98, not LAL 120. Given this evidence, I have opted to combine LAL 98 and 120 into 2MASS J03285216+3122453.

6. LAL 108 I strongly suspect to be identical with LAL 139, which is matched to 2MASS J03285521+3125223. LAL 139 itself does not seem to be offset; this must be the boundary between tiles in the original observation. The reported photometries for LAL 108 and 139 are nearly identical (matching at better than $0.08 \mathrm{mag}$, all bands).

7. I investigated whether it was reasonable to shift LAL 120 to become identical to LAL 147, because such a shift would be in the same direction. However, it is right on top of 2MASS J03285216+3122453, so I left it at that location. Confusingly, LAL 120 has photometry that is much different at $J$ from LAL 147 , but matches to within $0.13 \mathrm{mag}$ at $K$; LAL 120 and 2MASS J03285216 +3122453 have differing $J H K$ magnitudes by $>4$ mag. I have made no net changes as a result of this consideration.

8. LAL 124 moves from having no match to 2MASS $\mathrm{J} 03285630+3124432$. LAL do not report $J$ or $H$, but these sources match each other at $K$ to $0.13 \mathrm{mag}$.

9. LAL 134 I strongly suspect to be identical to LAL 165, which is well matched to 2MASS J03285842+3122567. The two LAL sources have no $J$ or $H$, but match each other in $K$ to $0.09 \mathrm{mag}$; they match the 2MASS $K_{s}$ to within $0.06 \mathrm{mag}$.

Just based on patterns, this seems to be the end of the sources I can (or need to) match in this region. Beyond the apparent corner of the tile, LAL 147, 166, 164, and 163 are all well matched to 2MASS counterparts. I cannot recover LAL 130; there is nothing at that location in 2MASS or IRAC, nor at a place offset in the same direction and size as the offsets above.

These coordinate uncertainties have some more minor repercussions in the rest of the catalog-there are things I strongly suspect are duplicates, as well as sources without counterparts that I can find. As for ASR, even given intrinsic variability, the LAL observations are not so deep as to be likely to reach sources undetected in the 2MASS, 2MASS $6 \times$, IRAC, or even the WISE observations. If there was no source in the 2MASS, IRAC, or WISE images at the LAL position or within a "reasonable" distance, I often dropped the LAL source; see Table 4.

In the end, I made changes to $37 \mathrm{LAL}$ sources. Sources that are duplicates are explicitly indicated in the last column in Table 2 via notation like "==LAL44" for LAL 38.

\subsection{X-Ray Catalogs}

Using a deep ROSAT HRI observation, Preibisch (1997) detected 20 X-ray sources, 16 of which were taken to be likely cluster members. Getman et al. (2002) report on a Chandra observation of this region, detecting 127 sources, 95 of which were identified with known cluster members. I retained only the source numbers (not the fluxes) reported in Preibisch (1997). Getman et al. (2002) find matches to all Preibisch sources except sources 1 and 2 . I was able to locate a counterpart at multiple other bands for source 1, and no counterpart at any band for source 2 . I suspect that source 2 from Preibisch (1997) may be spurious (or extragalactic), and I have removed it from my catalog.

Some of the sources from Getman et al. (2002) were specifically tagged as variable sources (in X-rays). No new YSO candidates were identified in their catalog, though it is quite likely many of the newly identified sources are members. They reported their entire catalog, which I absorbed into my database for the cross-IDs, though I did not retain the X-ray fluxes or luminosities in favor of our own re-reduction of the Chandra data (R14).

I found two inconsistencies in the Getman et al. (2002) cross-matching between this catalog and LAL and ASR, which I corrected in the catalog. Many of the cross-matches in Getman et al. (2002) are correct. However, LAL 79 is much closer to ASR 126 than 127, which is not what is reported in Getman et al. (2002). LAL 93 moved as per the discussion in Section 3.3 above; therefore, LAL 93 should not actually be matched to Getman source 15 . Getman source 15 should be matched to 2MASS J03270047+3137259.

Preibisch (2003) reports on an XMM-Newton observation of NGC 1333. It covers a wider area than the Chandra data from Getman et al., but most of the sources identified in this paper are also identified in Getman et al. There are seven sources identified based solely on the $X M M$ data. Preibisch finds counterparts in the optical or IR for five of the sources. Given the coordinates in Preibisch, two of the objects are within $1^{\prime \prime}$ of objects in my database, and the rest are within $2^{\prime \prime}$ of objects in my database, including the two claimed not to have a counterpart. I have made these associations in my catalog. Names from this study are incorporated into mine, including for the two objects previously claimed to not have counterparts.

The XMM-Newton Serendipitous Source Catalogue 3XMMDR4 was released in 2013 and consists of source detections over most of the XMM data taken as of 2012 December, which includes the NGC 1333 observations from Preibisch (2003). I find 46 matches to sources in this region, only 10 of which do not already have a Chandra detection in our reprocessed data. Names for these sources are included in the "other names" field, preceeded by "XMM."

There are no sources for which I now have an X-ray measurement (from YSOVAR's reprocessing or from 3XMMDR4) that did not already appear in Preibisch (1997, 2003), Getman et al. (2002), or Winston et al. (2009, 2010), described below.

\subsection{Rodríguez et al. (VLA) Catalog}

Rodríguez et al. (1999) report on Very Large Array (VLA) observations at 6 and $3.6 \mathrm{~cm}$ of an $8^{\prime} \times 8^{\prime}$ region centered on the HH 7-11 region. The nomenclature for this catalog as established in the paper is not "RAC" (as one might assume) but rather "VLA." Most of the objects were identified with a YSO counterpart in this paper; some were identified as variable. I retained nomenclature and positions from this catalog, because they are still in wide usage today. 
Table 4

Summary of LAL Modifications

\begin{tabular}{|c|c|}
\hline LAL Number & Notes \\
\hline 27 & Nothing at this location and nearby sources already have matches; removed \\
\hline 35 & Nothing at this location and nearby sources already have matches; removed \\
\hline 39 & $\begin{array}{l}\text { Nothing at this location and nearby sources already have matches; nearby bright source } 2 \text { MASS J03283695+3123121 does not have match, but that } \\
\text { is nearly an arcminute away and thus is an unlikely match; removed }\end{array}$ \\
\hline 44 & Nothing at this location and nearby source already has match; removed \\
\hline 47 & Nothing at this location, no nearby sources; removed \\
\hline 50 & Nothing at this location, and nearby sources already have matches; removed \\
\hline 52 & $\begin{array}{l}\text { This one, plus } 59 \text { and } 64 \text {, make an arc of } 3 \text { sources; this shape is not matched to anything in the 2MASS or IRAC images, and nearby sources have } \\
\text { matches; removed }\end{array}$ \\
\hline 59 & See 52; removed \\
\hline 61 & Near a faint $($ at $3.6 \mu \mathrm{m})$ extended source in [3.4] (WISE) and [3.6], but not in 2MASS; morphology suggests jet; removed \\
\hline 64 & See 52 ; removed \\
\hline 71 & Nothing at this location, no nearby sources; removed \\
\hline 81 & One of the " 54 " to the southeast," now 2MASS J03284764+3124061 \\
\hline 84 & Nothing at this location, no nearby sources; removed \\
\hline 85 & One of the " 54 " to the southeast," now 2MASS J03284947+3125066 \\
\hline 87 & One of the " $54 "$ to the southeast," now 2MASS J03284971+3124534 \\
\hline 93 & $\begin{array}{l}81 \text { moved close to original position of this one as part of the " } 54 \text { " to the southeast"; this one moves from 2MASS J03284764+3124061 (where it is } \\
\text { offset from a bright star, and to which it has sometimes been tied in the literature) to 2MASS J03285097+3123479 }\end{array}$ \\
\hline 98 & One of the " $54 "$ to the southeast," now identical to LAL 120, matched to 2MASS J03285216+3122453 \\
\hline 108 & One of the " 54 " to the southeast," now identical to LAL 139 , matched to 2MASS J03285521+3125223 \\
\hline 116 & Nothing at this location, and nearby sources already have matches; removed \\
\hline 118 & Nothing at this location, and several nearby sources all already have matches; removed \\
\hline 120 & $\begin{array}{l}\text { Could justifiably move to match LAL 147, but left it matched to 2MASS J03285216+3122453 (see " } 54 \text { " to the southeast" discussion); no net } \\
\text { changes }\end{array}$ \\
\hline 122 & Nothing at this location, and nearby sources already have matches; removed \\
\hline 124 & One of the "54" to the southeast," now 2MASS J03285630+3124432 \\
\hline 130 & Nothing at this location, nor at a place offset in the same direction and size as the " 54 " to the southeast"; removed \\
\hline 134 & One of the " 54 " to the southeast," now identical to LAL 165 , matched to 2 MASS J03285842+3122567 \\
\hline 135 & Nothing at this location, and nearby sources already have matches; removed \\
\hline 149 & In original LAL catalog, within $0 .{ }^{\prime \prime} 6$ of 148 , though photometry is different; retained 148 (and removed this one) \\
\hline 174 & Nothing at this location, and nearby sources already have matches; removed \\
\hline 242 & In original LAL catalog, within $00^{\prime \prime} 13$ of 241 , and only band available $(K)$ matches to 0.02 mag; retained 241 (and removed this one) \\
\hline 244 & Nothing at this specific location, but on edge of complex extended region with many sources; unclear what should match it; removed \\
\hline 259 & In original LAL catalog, 258 and 259 have identical positions and matching photometry; retained 258 (and removed this one) \\
\hline 271 & $\begin{array}{l}270-271 \text { are } 1 . \prime 03 \text { apart, and are likely two components of an object that is multiple in the 2MASS catalog, but both of the LAL sources are much } \\
\text { closer to 2MASS J03291433+3114441 than 2MASS J03291409+3114423; combined and } 270 \text { retained }\end{array}$ \\
\hline 281 & Nothing at this location and nearby source already has match; removed \\
\hline 284 & Nothing at this location and nearby sources already have matches; removed \\
\hline 289 & $\begin{array}{l}\text { In original LAL catalog, } 289 \text { and } 290 \text { have identical positions though different photometry (one is missing } J, H ; K \text { matches to } 0.02 \text { mag) identical to } \\
\text { 290; retained } 290 \text { (and removed this one) }\end{array}$ \\
\hline 291 & Nothing at this location and nearby sources already have matches; removed \\
\hline 338 & Nothing at this location and nearby source already has match; removed \\
\hline 352 & Nothing at this location and nearby sources already have matches; removed \\
\hline
\end{tabular}

Finding counterparts between 3.6 and $6 \mathrm{~cm}$ objects and NIR to mid-IR (MIR) sources can be quite complicated, as there is no assurance that the emitting source is the same. I have thus retained some VLA sources without shorter-wavelength counterparts, and I have shifted some sources to match shorter-wavelength counterparts. As mentioned above in Section 3.2, VLA 27 has been tied (including by Rebull et al. 2003) to one of the ASR sources. I have now tied VLA 27 to that source (R15-NGC 1333 J032911.24 $+311831.8=\mathrm{c} 2 \mathrm{~d} \mathrm{~J} 032911.3+311831)$, but not any of the ASR sources. Also, I have tied VLA 43 to ASR 114 (=2MASS $\mathrm{J} 03290149+3120208=\mathrm{R} 15-\mathrm{NGC} \quad 1333 \quad \mathrm{~J} 032901.53$ $+312020.6)$ and VLA 42 to 2MASS J03290116+3120244 (R15-NGC 1333 J032901.16+312024.4). Based on longwavelength information from Herschel (in the context of the Sadavoy et al. (2014) data incorporation below), I have associated VLA 28 with R15-NGC 1333 J032912.05 +311301.4 .

\subsection{Other NIR and MIR Data}

Rebull et al. (2003) reported on ground-based MIR data, which has largely been superceded by Spitzer data. However, the source cross-identifications and the source multiplicities found there are useful and have been retained in the catalog. For example, SVS 12 may be extended, and SVS 16 and ASR 107 are both multiple sources.

Aspin (2003) reported on NIR spectroscopy, including spectral types that I have included in my database. Some types are not particularly precise ("early K") but for many stars, this is all that is available.

Wilking et al. (2004) revisited the cluster with $J H K$ over a $\sim 11^{\prime} \times 11^{\prime}$ region to $K \sim 16$. They also searched for brown 
dwarfs, reporting on spectral types for their 25 candidates. The catalog abbreviation for this study, as established in their paper, is "MBO," standing for Mount Bigelow Observatory. In the published Wilking et al. (2004) catalog, there are two MBO 221 objects. Both have (separate) IRAC counterparts, only one has a 2MASS counterpart, and it is faint at that (it comes from the 2MASS $6 \times$ catalog). In consultation with B. Wilking (2015, private communication), the source on the west was manually added. MBO 222 is the highest number in the published catalog, so this source is now assigned to be $\mathrm{MBO}$ 223. Thus, MBO 221 is now matched to R15-NGC 1333 $\mathrm{J} 032847.19+311845.9$ and MBO 223 is now matched to R15NGC $1333 \mathrm{~J} 032847.27+312310.0$. For completeness, and for the benefit of future users of the catalog, I note that there are also several MBO numbers missing entirely.

\subsection{Cryo-era Spitzer Catalogs}

As noted above, the first Spitzer data for the NGC 1333 region were taken between the GTOs and the c2d Legacy team. The Legacy team mapped the entire Perseus molecular cloud complex, including NGC 1333; Jørgensen et al. (2006) reported on the IRAC data, and Rebull et al. (2007) reported on the MIPS data. Notably, the c2d data delivery included "bandfilled flux densities," meaning that if the source was not detected, an aperture was laid down at the location of the source to obtain an upper limit. I included flux densities and upper limits from the $\mathrm{c} 2 \mathrm{~d}$ catalog where measurements were not already present in our reprocessing of the cryogenic-era data. (This was the case where there was a low signal-to-noise ratio detection, or a limit.) Note that our cryo reprocessing does not include 70 or $160 \mu$ m fluxes; where relevant (rarely, in this very crowded region), I obtained $70 \mu \mathrm{m}$ measurements from the $\mathrm{c} 2 \mathrm{~d}$ catalog. The $\mathrm{c} 2 \mathrm{~d}$ project also identified YSO candidates using a multiband color selection; I retained in the database an indication of this status.

Gutermuth et al. (2008) reported on the Spitzer data specifically for NGC 1333 (as opposed to the entire Perseus cloud as c $2 d$ did). Only the candidate young stars were reported in Gutermuth et al. (2008). Since these cryogenic observations were reprocessed for YSOVAR using the same approach as Gutermuth et al. (2008), I only retained identifications of YSO candidates from Gutermuth et al. (2008).

Jørgensen et al. (2007) reported on submillimeter data combined with the Spitzer c2d data. They included a list of embedded YSOs in Perseus. These cross-IDs are included in "other names" as J07-Xx.

Arnold et al. (2012) included data from a Spitzer Infrared Spectrograph (IRS) survey of objects in NGC 1333. From this article, I have retained cross-IDs, SED classes (for comparison to ours), and identifications of YSOs (and non-members).

\subsection{Hatchell et al. (2007) Catalog}

Hatchell et al. (2007) observed Perseus at 450 and $850 \mu \mathrm{m}$. For some sources, they compiled SEDs and made crossidentifications. I included the cross-identifications in my catalog, as well as made new associations between these sources and the short-wavelength counterparts.

Source number 49 is listed as a match to an Infrared Array Satellite (IRAS) source, as well as the 2MASS source J03283609+3113346. Looking at the 2MASS and WISE images, this is an extended source, and that 2MASS source is one of three near the core of it. The 2MASS point source closest to the core of the extended source is J03283681 +3113326 ; the 2MASS extended source catalog source to which it should be matched is $\mathrm{J} 03283630+3113329$.

Source number 70 does not have a short-wavelength counterpart listed in Hatchell et al. There is nothing obvious there in the 2MASS images, and only faintly is there a source in the WISE images. There is a source from Spitzer/IRAC at J032914.96+312031.7, which is 4!" 4 away from the position given for source 70, within the range of positional uncertainties given by the Hatchell et al. cross-references to 2MASS. Hatchell et al. place this source as an SED Class 0 . I have very few points between 2 and $25 \mu \mathrm{m}$ delineating J032914.96 +312031.7 - I only have four IRAC bands, and it is falling, not rising. I place it in SED Class II. The Hatchell et al. source 70 may not be a good match to the source at R15-NGC 1333 $\mathrm{J} 032914.96+312031.7$, but it is the best thing I can match to it at this time. A note about this uncertainty is included in the last column of Table 2 .

\subsection{Brown Dwarf Searches}

There are several studies seeking brown dwarfs in this region. Oasa et al. (2008) is one of these brown dwarf searches, using $J H K_{s}$ over a $\sim 5^{\prime} \times 5^{\prime}$ region with a spatial resolution of $\sim 1$."5. Their YSO candidate identifications were retained in my catalog. Matches were found within $2^{\prime \prime}$ for $85 \%$ of their sources. The rest of the sources were retained in the catalog with the positions from Oasa et al., except for one, which I now describe.

I find that one of the sources reported upon in Oasa et al. (2008) is subject to source confusion issues. It is in the region associated with IRAS-2 b. The source in question was assigned a position of $03: 28: 57.09,+31: 14: 21.4$ by Oasa et al., who reported no other sources within $40^{\prime \prime}$ of this location. In the IRAC images, two sources can be seen, J032857.37+311415.7 and $\mathrm{J} 032857.20+311419.1$, both of which are quite far away from the source from Oasa et al. (7". 4 and 3". 4, respectively). They have very different flux ratios-the more northern one dominates at $\sim 3 \mu \mathrm{m}$ (and is closer to the original Oasa position), but by $\sim 8 \mu \mathrm{m}$, the southern one dominates. By inspection of the available images (2MASS, IRAC, and WISE), I conclude that this Oasa et al. source should not be retained as a separate source, but instead should be tied to one of the other two seen in the IR. Based on proximity, I have tentatively tied this source to R15-NGC 1333 J032857.20+311419.1. However, Oasa et al. report that this source is a Class III, and not a YSO. This is not consistent with the rest of the information I have on J032857.20+311419.1, but there are not any closer sources bright in the NIR. This is the best association I can make. A note about this uncertainty is included in the last column of Table 2 .

Greissl et al. (2007) used the HST NICMOS instrument to search for brown dwarfs in NGC 1333. They reported $J H$ photometry and spectral types from low-resolution grism spectroscopy for their objects of interest, both of which I included in the database. Greissl et al. source S1-6 is very close to other sources in the region; Figure 5 shows the region in $3.6 \mu \mathrm{m}$. There is an "appendage" off of a brighter source; that "appendage" is what I matched to source S1-6=J032857.41 +311536.9 . The net SED for this source is largely optical/NIR data, from Greissl et al.; it is hard to apportion IR data correctly 


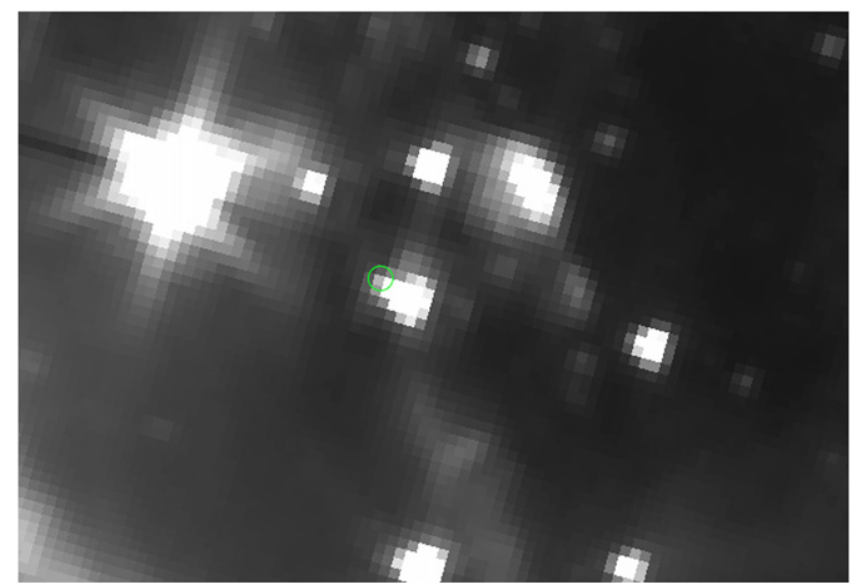

Figure 5. Region of sky in $3.6 \mu \mathrm{m}$ immediately around J032857.41 $+311536.9=$ Greissl S1-6, circled. North is up. The distinctive triangularly shaped IRAC point-spread function of the point sources can be seen in several point sources in this field of view, but one has an "appendage" extending to the left; the "appendage" is matched to Greissl S1-6.

for this source from telescopes other than HST. I considered that this source may be incorrectly matched to an IR source, and that the optical points may correspond to a different source. The nearest one I can identify is the brighter source in Figure 5 (J032857.15+311534.6), which is $\sim 4^{\prime \prime}$ away; this is significantly larger than the IR positional uncertainties, or that of Greissl et al., so this is unlikely. Moreover, since they were working with HST data, it is likely that their astrometry is correct. I have left it in the catalog asis. There are notes about this in the last column of Table 2 for $\mathrm{S} 1-6=\mathrm{J} 032857.41$ +311536.9 .

Scholz et al. (2009, 2012a, 2012b) are all part of the SONYC (Substellar Objects in Nearby Young Clusters) survey searching for brown dwarfs. These investigators obtained very deep imaging in four bands $\left(i^{\prime}, z^{\prime}, J, K\right)$ but only report photometry for their brown dwarfs, candidates, and rejects, which I incorporate into the catalog. I also included the spectral types and membership as reported in the SONYC papers, both for stellar and substellar objects. Scholz et al. (2012a) report a new very low mass member, which becomes $S-45$. However, it is within $0.2^{\prime \prime}$ of $\mathrm{Sp} 105$, a source they list as a previously confirmed member in Scholz et al. (2012b). I take these two to be referencing the same object. They have essentially identical NIR magnitudes and spectral types in the papers.

Itoh et al. (2010) is another search for brown dwarfs; spectral types and cross-identifications from this paper were incorporated into the database.

\subsection{Winston Catalog}

Winston et al. $(2009,2010)$ report on a combined Spitzer and Chandra investigation of NGC 1333. Follow-up spectra were also included. A full X-ray catalog (of every source detected) was included in Winston et al. (2010). Spectral types and X-ray identifications were incorporated from this paper into my database, as well as whether Winston et al. identified the object as a YSO. The X-ray measurements from Winston et al. were superceded by our own re-reduction (R14).

The source numbers presented in Winston et al. (2009) refer to source numbers tied to coordinates (R.A./decl.) in Winston et al. (2010), but there is some potential confusion as to whether the numbers are sequential IR or X-ray numbers, where the former come from Gutermuth et al. (2008) and the latter are assigned in the Winston work. The numbers in Winston et al. (2009) are not specified as being IR or X-ray numbers. Winston et al. (2010) report both X-ray and IR numbers, but not all of them appear in Gutermuth et al. (2008). Via personal communication with E. Winston, I have verified that the numbers in Winston et al. (2009) are meant to be IR numbers. The IR numbers in Winston et al. $(2009,2010)$ that are greater than 137 do not appear in Gutermuth et al. (2008). These are ones that Winston et al. assigned (not Gutermuth et al.) and are defined via postions (R.A./decl.) in Winston et al. (2009). Thus, while Gutermuth et al. (2008) only assigns numbers to YSO candidates reported there, there can be a "Gutermuth08 number" assigned by Winston et al. (2009, 2010). In other words, Winston et al. (2009) report those as YSO candidates selected from the IR, but Gutermuth et al. (2008) do not report those objects as YSO candidates. All of this has been resolved in my catalog.

\subsection{Sadavoy et al. Herschel Identifications}

Sadavoy et al. (2014) report on candidate Class 0 objects in NGC 1333 using Herschel data. Because the shortest Herschel wavelength, $70 \mu \mathrm{m}$, overlaps with the longest Spitzer wavelength I have here (also $70 \mu \mathrm{m}$ ), I wished to link the Herschel observations with the rest of my catalog. However, this is another example of the difficulty in finding clear correspondences between sources at shorter and longer wavelengths. In many cases, the corresponding source at 3.6 and/or $4.6 \mu \mathrm{m}$ is blended with nebular emission and may not represent flux solely from the source seen at long wavelengths. Some of the sources in Sadavoy et al. in this region are noted as not having Spitzer counterparts, but I have been able to find counterparts for most of them. All of these cross-identifications appear as part of the "other names" in Table 2; where the association is uncertain, a question mark is added to the cross-identification. No flux densities are reported in Sadavoy et al. (2014), so these points cannot be added to tables or SEDs. My assembled SEDs for these objects appear in Figure 6. Notes on specific sources follow.

Counterparts to Sadavoy et al. sources West162 and West33 are likely to have contamination from extended emission at 3.6 and $4.5 \mu \mathrm{m}$. Moreover, for West33, the short-wavelength counterpart has a falling SED, but by $24 \mu \mathrm{m}$, it is very bright and rising fast. The source detected at 3-8 $\mu \mathrm{m}$ may not be the same object seen at $>24 \mu \mathrm{m}$ in this case.

West19 is very complicated because it is in a very bright and confused region. There does seem to be an object (J032901.91 +311541.4 ) at IRAC wavelengths close to that location; at 7".5, it is among the largest positional offsets for all of the matches to the Sadavoy sources, but consistent with the postional uncertainties. At $24 \mu \mathrm{m}$, the object at this location is very unfortunately placed in the MIPS image so as to be largely obscured by the Airy ring of a nearby extremely bright source. (Note that I have not tied West19 to the very bright $24 \mu \mathrm{m}$ source, since it is too far away!) This association is the best I can do at this time, despite the relatively large positional offset.

West40 and West87 are both in the same neighborhood as West19, though they are both farther from the very bright sources and extended emission that plague West19. By contrast, West40's match to J032904.07+311446.5 seems relatively straightforward, though the $J$-[24] SED is somewhat unusually shaped. West87's match at J032906.45+311534.4 

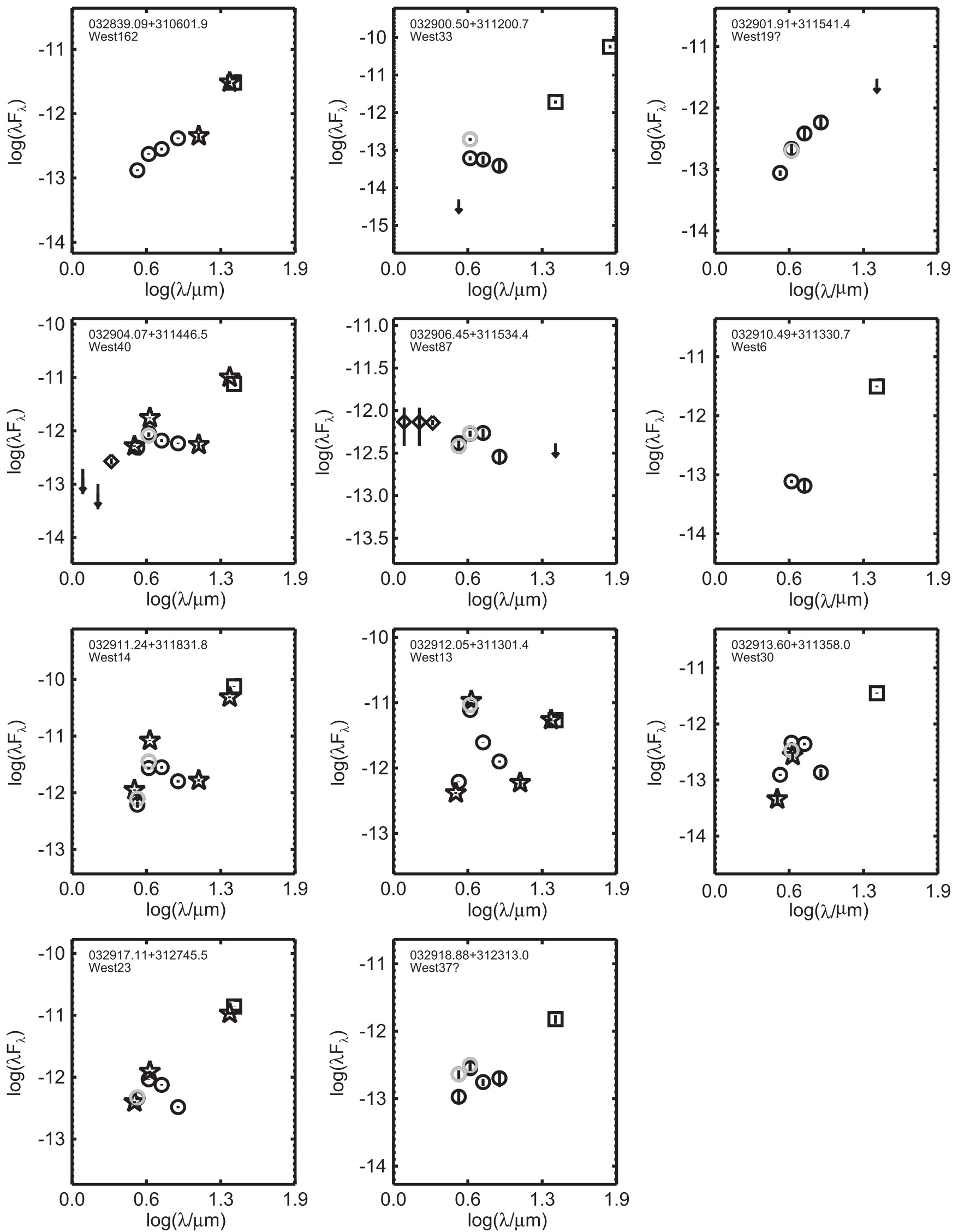

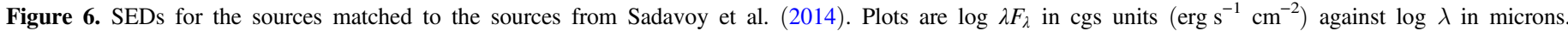

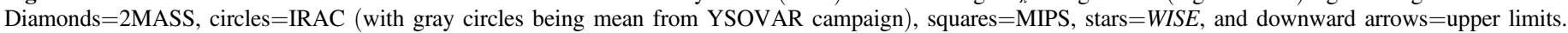

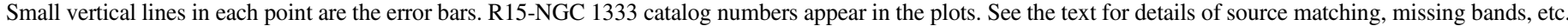


does not have a [24] measurement because of the halo around the bright $24 \mu \mathrm{m}$ source. It has another strange SED, and the object responsible for the emission at the shortest wavelengths may very well not be the same as for the $>20 \mu \mathrm{m}$ wavelengths. Because it is missing SED points longer than $20 \mu \mathrm{m}$, and because of the $2-8 \mu \mathrm{m}$ slope, I place this object in SED Class II.

For West6, there is a clear correspondence of the $24 \mu \mathrm{m}$ source (at J032910.49+311330.7) and the Herschel source. However, no clear $8 \mu \mathrm{m}$ source can be seen on the IRAC image (and no $12 \mu \mathrm{m}$ source can be seen on the WISE image). There is a faint source at $4.5 \mu \mathrm{m}$, and perhaps something on the $5.8 \mu \mathrm{m}$ image, but nothing clear on the $3.6 \mu \mathrm{m}$ image. It is not apparent whether the source at 4.5 (and 5.8) $\mu \mathrm{m}$ is the same as the $24 \mu \mathrm{m}$ source and West6, but all measurements have been retained in this associated source.

West14 is in another very complicated region. There are several sources in close proximity, as well as extended nebular emission. I have tied it to R15-NGC 1333 J032911.24 +311831.8 on the basis of image morphology at Spitzer bands, but note that there could be contributions from other adjacent sources at nearly all bands. (For example, WISE may blend the two closest sources.)

West13 is another object that is difficult to match to shortwavelength sources. The $70 \mu \mathrm{m}$ source seen by Herschel has a clear counterpart in the $24 \mu \mathrm{m}$ MIPS images. However, there may be more than one source in the IRAC images at this location. Based on the images, I associate West13 with R15NGC 1333 J032912.05+311301.4 as the most likely match. Based on this association, I move VLA 28 from its nominal published location to coincide with this source as well. There is a nearby source seen in 2MASS $6 \times$, but only at $K_{s}$. This source, however, is too far away to be associated with R15-NGC 1333 J032912.05+311301.4, so I do not associate them.

West 30 and West 23 are both additional cases of a clear match among the $70,24,8$, and $5.8 \mu \mathrm{m}$ images, but the measurements at 3.6 and $4.5 \mu \mathrm{m}$ may be affected by extended emission.

West 37 is another very complicated, bright region. There is clearly an object in the $3.6,4.5$, and $5.8 \mu \mathrm{m}$ images, but if I had only those short wavelengths, I might have called it a dust clump based on image morphology. There is a source faintly seen in the $24 \mu \mathrm{m}$ image, but it is on a nearby source's Airy ring. I have provisionally tied West37 to R15-NGC 1333 $J 032918.88+312313.0$.

\subsection{Foster APOGEE Data}

Foster et al. (2015) report on the velocity dispersion of young stars in NGC 1333 based on NIR spectra obtained with the Apache Point Observatory Galactic Evolution Experiment (APOGEE; Zasowski et al. 2013). Target selection for that project included considerations based on the variability of objects in the YSOVAR data, so many of the objects in which we are interested in the context of YSOVAR also have measurements in Foster et al. Through spectral fitting, Foster et al. derive several parameters for stars with sufficient signalto-noise ratios in their APOGEE spectra, including $T_{\text {eff }}, \log g$, and $v \sin i$. I included in my database cross-identifications, identifications of non-members, $T_{\mathrm{eff}}$, and $v \sin i$, as well as other information from that paper.

\subsection{YSOVAR Data}

Because I had originally undertaken this project in the context of analysis of our YSOVAR data, I have taken the liberty in this paper of including the means from those light curves (at 3.6 and $4.5 \mu \mathrm{m}$ ) in my database. As mentioned above, the NGC 1333 YSOVAR data will be described in detail in L. M. Rebull et al. (2015, in preparation).

\subsection{Two Very Bright, Confused Sources}

In one of the brightest regions of NGC 1333, there are two sources very close to each other, at or near the spatial resolution of many of the surveys here. Early on, this pair of sources was identified as SVS 12=IRAS 6. However, in 2MASS, IRAC, and even in MIPS images, two sources can be distinguished by eye if the stretch is severe enough. Both of these objects have slightly different SEDs, certainly in part due to saturation and flux apportionment issues. The one tagged ASR 114=LAL $181=$ MBO $19=$ Gutermuth $27=\mathrm{c} 2 \mathrm{~d}$ J032901.6 $+312021=\mathrm{R} 15$ NGC 1333 J032901.53+312020.6 made it onto most published lists as a YSO or candidate. For reasons probably due to saturation and/or spatial resolution and/or flux apportionment, the second did not; it is c2d J032901.2+312025=R15-NGC $1333 \mathrm{~J} 032901.16+312024.4$. In the c2d catalog, one of these sources gets all the MIPS $70 \mu \mathrm{m}$ flux density, though the other source probably should be allocated some of it. Many bands are listed as limits for these sources in various catalogs; they are probably meant as lower, not upper, limits, though they are often tagged upper limits. I have declared both of these sources to be literature-identified YSOs and added a note about the $70 \mu \mathrm{m}$ flux apportionment to the last column in Table 2.

\subsection{Jet Drivers}

I consulted two papers to identify the sources most commonly thought of as driving outflows and/or jets in NGC 1333. Davis et al. (2008) used $2.122 \mu \mathrm{m}$ imaging to identify outflows; they identified 11 sources as having outflows of any sort, and their IDs were tied back to those from Jørgensen et al. (2007). Plunkett et al. (2013) reported on CARMA observations of outflows, identifying seven YSOs as the drivers of the outflows. Three sources are identified by both papers, resulting in 12 sources identified as driving jets or outflows. These sources, as well as some of their other names, are included in Table 5.

\subsection{Studies That Are Not Included}

There are several individual famous objects of particular interest in this region, some with many papers entirely of their own (e.g., the components that make up IRAS-2, IRAS-4, ...). I did not include additional data from these many individual projects in my catalog, but instead assume that users can match by cross-ID to any few sources of interest (though I encourage image inspection via the IRSA tool FinderChart if nothing else). I also did not place an emphasis on matching to many surveys at wavelengths longer than about $50 \mu \mathrm{m}$, because (a) there are relatively few true point sources at the longer bands, and (b) it is often hard to ensure a good match between the point sources from shorter wavelengths and those at the longer wavelengths. Some long-wavelength matches have been made in three cases - the VLA catalog is the source of some nomenclature still in wide use, and both the Hatchell et al. 
Table 5

List of Jet/Outflow Driving Sources

\begin{tabular}{|c|c|c|}
\hline Our ID (R15-NGC $1333 \mathrm{~J})$ & Reference(s) & Some of the Other IDs \\
\hline $032837.06+311331.0$ & Davis et al. (2008) & IRAS1, IRAS 03255+3103, J07-11 \\
\hline $032845.30+310542.0$ & Davis et al. (2008) & IRAS $03256+3055$, J07-14 \\
\hline $032855.53+311436.3$ & Plunkett et al. (2013), Davis et al. (2008) & IRAS 2 A, SK8, J07-15 \\
\hline $032857.37+311415.7$ & Davis et al. (2008) & IRAS 2a, J07-16 \\
\hline $032900.50+311200.7$ & Plunkett et al. (2013), Davis et al. (2008) & IRAS 4B1, SK1, Sadavoy2014-West33, J07-18 \\
\hline $032903.39+311602.0$ & Davis et al. (2008) & J07-20 \\
\hline $032903.75+311603.9$ & Plunkett et al. (2013) & SVS13, IRAS3, IRAS 03259+3105, \\
\hline $032904.07+311446.5$ & Plunkett et al. (2013) & IRAS5, Sadavoy2014-West40, SK14, J07-21 \\
\hline $032910.49+311330.7$ & Plunkett et al. (2013), Davis et al. (2008) & IRAS4A, SK4, Sadavoy2014-West6,J07-22 \\
\hline $032910.70+311820.9$ & Davis et al. (2008) & J07-23 \\
\hline $032911.24+311831.8$ & Davis et al. (2008) & Sadavoy2014-West14,J07-24 \\
\hline $032912.05+311301.4$ & Plunkett et al. (2013) & IRAS 4B, SK3, Sadavoy2014-West13, J07-25 \\
\hline
\end{tabular}

(2007) and Sadavoy et al. (2014) papers made specific efforts to find matches at the shorter wavelengths. A comprehensive set of matches to all of the submillimeter and millimeter sources in the literature is beyond the scope of this paper.

\section{OBVIOUS GAPS FOR FUTURE WORK}

About half of the objects in this region that are identified as YSO candidates lack spectroscopy. This could be used not just for spectral classification, but for confirmation that they are young stars, or even just confirmation that they are not background galaxies (this is a very real concern; see, e.g., Rebull et al. 2010). Most of the spectral types found in the literature come from searches for brown dwarfs, so the set of objects for which there are spectral types is incomplete and highly skewed to late $\mathrm{M}$ and later. There are about 150 objects (out of $\sim 300$ YSO candidates) that have some sort of estimate of spectral type in the literature, even a coarse one (e.g., " $<\mathrm{M} 0$ " or "early K"). The $T_{\text {eff }}$ values from Foster et al. (2015) can be used to constrain the spectral type, even though the $T_{\text {eff }}$ values are much more uncertain for the hotter stars. However, there are only about 20 objects for which there is a $T_{\text {eff }}$ estimate but no published spectral type of any kind. Spectroscopy would help limit the non-member contamination and improve the inventory of members (and therefore knowledge of the mass function) in NGC 1333.

Multi-band broadband optical data could be very helpful over this entire region for delineating the Wien side of the SED. Even just $r$ and $i$ (or similar) would help show if the SEDs are, in fact, turning over for the YSO candidates. Moreover, short wavelengths such as $u$ - and $g$-band data would be useful for constraining mass accretion rates in these young stars, but such observations would be quite challenging due to the high extinction toward most sources. High spatial resolution optical observations have been shown (e.g., Rebull et al. 2010) to be critical for distinguishing background star-forming galaxies from nearby YSOs, since both kinds of objects overlap in IR color space with IR colors indicating star formation.

X-ray data can be very helpful for identifying young stars without disks. The existing Chandra and XMM data are limited in area, focused just on the heart of NGC 1333. X-ray data over a larger area could help identify additional less embedded cluster members.

Some of the most famous YSOs in this region are those originally identified by IRAS in 1983 . However, most of these very bright sources have broken into pieces every time astronomers have looked with higher spatial resolution. Some sources are still clearly multiple and still have flux apportionment problems. Higher spatial resolution MIR and far-IR observations will aid in correctly attributing source flux to the correct source. In some cases, the surface brightness is so high that higher spatial resolution observations could be very difficult.

\section{PLACEMENT INTO SED CLASSES}

After assembling all of the literature data, including the mean [3.6] and [4.5] measurement from our YSOVAR campaign, I constructed an SED for each object using all available data. Some objects have well-defined SEDs, with data from optical to 8,24 , or even $70 \mu \mathrm{m}$; others have far less well defined SEDs, for example, with only one point that is the mean of one channel's light curve from our YSOVAR campaign.

As discussed in R14, I defined an internally consistent placement of the YSOVAR objects into SED classes as follows. In the spirit of Wilking et al. (2001), I define the NIR to $\operatorname{MIR}(2-24 \mu \mathrm{m})$ slope of the SED, $\alpha=d \log \lambda F_{\lambda} / d \log \lambda$, where $\alpha>0.3$ for a Class I, 0.3 to -0.3 for a flat-spectrum source, -0.3 to -1.6 for a Class II, and $<-1.6$ for a Class III. For each of the YSOs and candidate YSOs in the sample, I performed a simple ordinary least-squares linear fit to all available photometry (just detections, not including upper or lower limits) as observed between 2 and $24 \mu \mathrm{m}$, inclusive. Note that formal errors on the IR points are generally so small as to not affect the fitted SED slope. Note also that the fit is performed on the observed SED, e.g., no reddening corrections are applied to the observed photometry before fitting. Classification via this method is provided specifically to enable comparison to other YSOVAR papers via internally consistent means; see discussion in R14. I can only perform this calculation for those objects with points at more than one wavelength in their SED between 2 and $24 \mu \mathrm{m}$. Therefore, objects for which I have, e.g., one $3.6 \mu \mathrm{m}$ point from the cryogenic era and one $3.6 \mu \mathrm{m}$ YSOVAR point (the mean YSOVAR measurement at this band) cannot have a fitted SED slope.

The SED slopes and classes I calculate appear in Table 3. Slopes can be calculated for any of the objects with at least two distinct wavelengths in the SED between 2 and $24 \mu \mathrm{m}$, but it is only meaningful if the source is a YSO, so I only include slopes and a class for objects tagged as YSOs by any of the references I included in this paper. Of the $\sim 300$ objects in the catalog that 


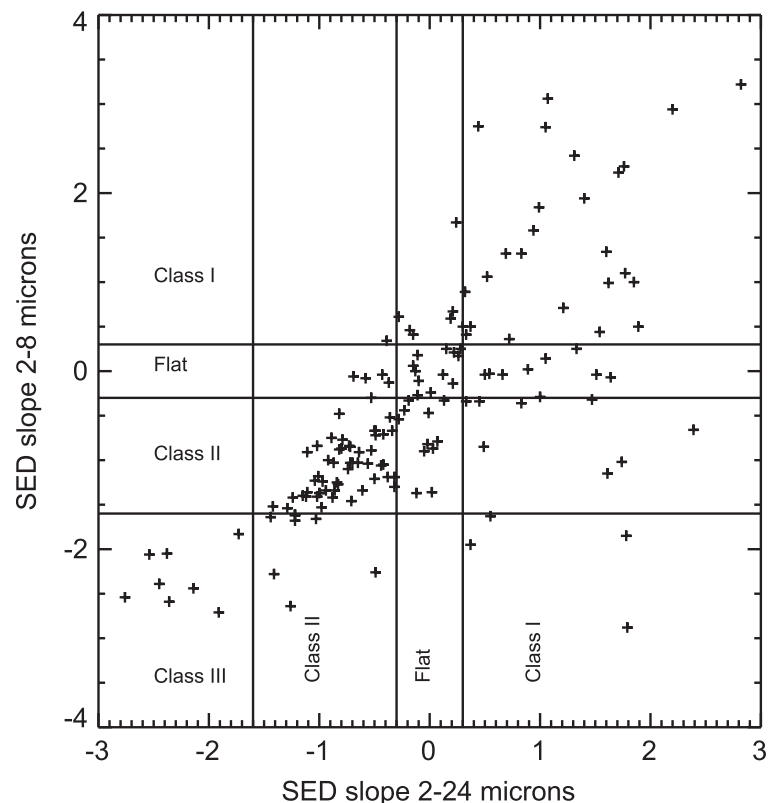

Figure 7. Comparison of the SED slopes calculated from the observed SED, just for those objects with $24 \mu \mathrm{m}$ detections and identified somewhere in the literature as YSOs. Since the $24 \mu \mathrm{m}$ detections are strongly biased toward cluster members, most of the points here are YSOs (or candidates). The vertical and horizontal lines indicate the divisions between SED classes as defined in Section 5 . For $65 \%$ of the entire set of objects, the resulting SED class is the same. The objects that do not match typically have a borderline slope. As expected, there is a bias such that inclusion of the $24 \mu \mathrm{m}$ point frequently pushes an object to more embedded SED classes.

are tagged as YSOs, where I can calculate the slope between 2 and $24 \mu \mathrm{m}$, I find 55 Class Is, 38 Flat class, 103 Class IIs, and 81 Class IIIs. There are 25 with insufficient points in the SED, such that I cannot assign a class.

In R14, we asserted that the classes are generally well matched whether or not the $24 \mu \mathrm{m}$ point is included. To understand the influence of the $24 \mu \mathrm{m}$ point, in the cases where there is a $24 \mu \mathrm{m}$ point, I can compare the $2-24$ and $2-8 \mu \mathrm{m}$ SED slopes. Figure 7 compares the fitted slope for the 2-24 and 2-8 $\mu \mathrm{m}$ approaches, just for those sources detected at $24 \mu \mathrm{m}$ and also identified as candidate YSOs. (The $24 \mu \mathrm{m}$ detections are strongly biased toward cluster members, so this figure includes most of the $24 \mu \mathrm{m}$ detections.) The vertical and horizontal lines indicate the divisions between SED classes as defined above. For $\sim 65 \%$ of this subset of objects, the resulting SED class is the same. (Table 6 has the total numbers.) The objects that do not match typically have a borderline slope. As expected, there is a bias such that inclusion of the $24 \mu \mathrm{m}$ point frequently pushes an object to more embedded SED classes. There are a few sources that are approximately photospheric until a sharp rise at $24 \mu \mathrm{m}$; those are difficult to classify correctly using our approach. Of the $\sim 300$ objects identified as YSOs (or candidates), $\sim 150$ are detected at $24 \mu \mathrm{m}$, and $\sim 100$ have the same class even including the $24 \mu \mathrm{m}$ point.

In R14, we also asserted that our classes are generally well matched to the G09 classes. Arnold et al. (2012) also report classes for YSO candidates. Table 6 has the numbers of objects in my catalog for which my classes match (or do not match) the classes obtained from these other approaches. The first thing to notice is that both G09 and Arnold et al. (2012) have different class bins than I have defined-G09 has no flat class, and a "II/ III" class, and Arnold et al. have transition disk (TD) and pretransition disk (PTD) classes. Despite this, the majority of the sources have the same class regardless of approach.

For the most embedded sources, there is no provision in our scheme for identifying Class $0 \mathrm{~s}$, and I do not use points at wavelengths longer than $24 \mu \mathrm{m}$ to determine classes. I would expect, though, that those objects that others have identified as Class 0s would work out to be Class Is in my scheme. There are 10 sources with SED classifications from the submillimeter in Hatchell et al. (2007) for which I have counterparts-in Hatchell et al., eight of them are Class 0 and two are Class I, and by my classification, nine are Class I and one is Class II. There are 11 Class 0 sources in the region identified in Sadavoy et al. (2014) from Herschel data-out of those 11, all but one are Class I, but that last one is Class II. Both of those discrepant sources (J032914.96+312031.7 for the Hatchell source and J032906.45+311534.4 for the Sadavoy source) have been discussed above. The matches may not be a good match to the source of the long-wavelength flux. At any rate, in most cases, my method at least recovers Class I status for these very embedded objects.

Ideally, one would have a fully populated SED, as well as a spectral type, and thus be able to attempt SED modeling to determine the proper classes for these objects, but this is not possible at this time.

Table 6

Comparison of Classes

\begin{tabular}{|c|c|c|c|c|}
\hline Other Class & Our Class I & Our Flat Class & Our Class II & Our Class III \\
\hline Literature tagged YSOs: $2-8 \mu \mathrm{m}$ Class I & 25 & 7 & 1 & 0 \\
\hline Literature tagged YSOs: $2-8 \mu \mathrm{m}$ Flat Class & 9 & 12 & 5 & 0 \\
\hline Literature tagged YSOs: $2-8 \mu \mathrm{m}$ Class II & 8 & 11 & 49 & 0 \\
\hline Literature tagged YSOs: $2-8 \mu \mathrm{m}$ Class III & 4 & 0 & 8 & 8 \\
\hline Any matches: G09 Class I,I* & 29 & 9 & 0 & 0 \\
\hline Any matches: G09 Class II,II* & 5 & 19 & 67 & 3 \\
\hline Any matches: G09 Class II/III & 0 & 0 & 4 & 0 \\
\hline Any matches: Arnold12 Class I & 12 & 2 & 0 & 0 \\
\hline Any matches: Arnold12 Class FS & 2 & 7 & 2 & 0 \\
\hline Any matches: Arnold12 Class II & 0 & 1 & 33 & 0 \\
\hline Any matches: Arnold12 Class III & 0 & 0 & 0 & 1 \\
\hline Any matches: Arnold12 Class PTD & 0 & 1 & 3 & 0 \\
\hline Any matches: Arnold12 Class TD & 0 & 2 & 3 & 0 \\
\hline
\end{tabular}




\section{SUMMARY}

I have presented a catalog of apparent point-source objects toward NGC 1333 , within $52^{\circ}<$ R.A. $<52^{\circ} .5$ and $31^{\circ}<$ decl. $<31$. 6 . I have attempted to resolve a wide variety of confusion in the literature, ranging from mismatched sources to missing or duplicate IDs. I incorporated data from optical to radio wavelengths, but focused most of my effort on $J(1.25 \mu \mathrm{m})$ to $24 \mu \mathrm{m}$. Cross-identifications include those from more than 25 papers and catalogs from 1994 to 2015.

I have also identified obvious holes in the accumulated literature, the most obvious one being spectroscopic confirmation of the many YSO candidates presented in the literature, along with spectral types.

While I have done the best that I can, the reliability of this catalog is likely lower for the longer wavelengths and for the brightest and/or most confused regions in the heart of NGC 1333. Image inspection proved invaluable in making many of these associations.

I have compared a few different methodologies for classifying objects by the SED shape and/or IR colors. While the methods agree in most cases, they can fail in identifying the most embedded sources and sources that are on the borderline between SED class slope definitions, and they can more weakly depend on whether or not there is a detection at $\sim 20-25 \mu \mathrm{m}$ to anchor the SED slope between 2 and $24 \mu \mathrm{m}$.

We will use this catalog as the basis for our upcoming work using YSOVAR data in NGC 1333.

Special thanks to all of the authors of past studies who patiently answered my questions, dug up old data and notes, and helped me sort out which object was which. Thanks to David Shupe and the NASA-Herschel Science Center helpdesk for a quick processing of PACS and SPIRE images to help resolve source matching issues. Thanks to Jesus Hernandez for pointing out the problem between WISE All-Sky and AllWISE for the sources in IRAS 7 and to Chris Gelino for helping resolve it. Thanks to John Stauffer, Moritz Günther, and Lynne Hillenbrand for comments on the manuscript.

This research has made use of the NASA/IPAC Infrared Science Archive (IRSA), which is operated by the Jet Propulsion Laboratory, California Institute of Technology, under contract with the National Aeronautics and Space Administration. This work is based in part on observations made with the Spitzer Space Telescope, which is operated by the Jet Propulsion Laboratory, California Institute of Technology under a contract with NASA. Support for this work was provided by NASA through an award issued by JPL/Caltech. This research has made use of NASA's Astrophysics Data System (ADS) Abstract Service and of the SIMBAD database, operated at CDS, Strasbourg, France. This research has made use of data products from the Two Micron All-Sky Survey (2MASS), which is a joint project of the University of Massachusetts and the Infrared Processing and Analysis Center, funded by the National Aeronautics and Space Administration and the National Science Foundation. The 2MASS data are served by the NASA/IPAC Infrared Science Archive, which is operated by the Jet Propulsion Laboratory,
California Institute of Technology, under contract with the National Aeronautics and Space Administration. This publication makes use of data products from the Wide-field Infrared Survey Explorer, which is a joint project of the University of California, Los Angeles, and the Jet Propulsion Laboratory/ California Institute of Technology, funded by the National Aeronautics and Space Administration.

\section{REFERENCES}

Arnold, L. A., Watson, D., Kim, K., et al. 2012, ApJS, 201, 12

Aspin, C. 2003, AJ, 125, 1480

Aspin, C., Sandell, G., \& Russell, A. 1994, A\&AS, 165, 106

Bally, J., Walawender, J., Johnstone, D., Kirk, H., \& Goodman, A. 2008, in Handbook of Star Forming Regions, Vol. 1, ed. B. Reipurth (San Francisco, CA: ASP), 308

Davis, C. J., Scholz, P., Lucas, P., Smith, M. D., \& Adamson, A. 2008, MNRAS, 387, 954

Evans, N., Allen, L. E., Blake, G., et al. 2003, PASP, 115, 965

Evans, N., Dunham, M. M., Jørgensen, J., et al. 2009, ApJS, 181, 321

Fazio, G., Hora, J. L., Allen, L. E., et al. 2004, ApJS, 154, 10

Foster, J., Cotaar, M., Covey, K., et al. 2015, ApJ, 799, 136

Fruscione, A., McDowell, J. C., Allen, G. E., et al. 2006, Proc. SPIE, 6270, 1 Getman, K., Feigelson, E., Townsley, L., et al. 2002, ApJ, 575, 354

Greissl, J., Meyer, M., Wilking, B., et al. 2007, AJ, 133, 1321

Gutermuth, R., Megeath, S. T., Myers, P., et al. 2009, ApJ, 184, 18

Gutermuth, R., Megeath, S. T., Myers, P., et al. 2010, ApJS, 189, 352

Gutermuth, R., Myers, P. C., Megeath, S. T., et al. 2008, ApJ, 674, 336

Hatchell, J., Fuller, G., Richer, J., Harries, T., \& Ladd, E. 2007, A\&A, 468, 1009

Herbig, G., \& Jones, B. 1983, AJ, 88, 1040

Hirota, T., Bushimata, T., Choi, Y. K., et al. 2008, PASJ, 60, 37

Hirota, T., Honma, M., Imai, H., et al. 2011, PASJ, 63, 1

Itoh, Y., Gupta, R., Oasa, Y., et al. 2010, PASJ, 62, 1149

Jørgensen, J., Harvey, P., Evans, N. J., et al. 2006, ApJ, 645, 1246

Jørgensen, J., Johnstone, D., Kirk, H., \& Myers, P. 2007, ApJ, 656, 293

Karchenko, N., Piskunov, A., Schilback, E., Röser, S., \& Scholz, R. 2013, A\&A, 558, 43

Lada, C., Alves, J., \& Lada, E. 1996, AJ, 111, 1964

Oasa, Y., Tamura, M., Sunada, K., \& Sugitani, K. 2008, AJ, 136, 1372

Padgett, D., Rebull, L., Stapelfeldt, K., et al. 2008, ApJ, 672, 1013

Plunkett, A., Arce, H., Corder, S., et al. 2013, ApJ, 774, 22

Preibisch, T. 1997, A\&A, 690, 324

Preibisch, T. 2003, A\&A, 401, 543

Raga, A., Noriega-Crespo, A., Carey, S., \& Arce, H. 2013, AJ, 145, 28

Rebull, L., Cody, A., Covey, K., et al. 2014, AJ, 148, 92

Rebull, L., Cole, D., Stapelfeldt, K., \& Werner, M. 2003, AJ, 125, 2568

Rebull, L., Padgett, D., McCabe, C.-E., et al. 2010, ApJS, 186, 259

Rebull, L., Stapelfeldt, K., Evans, N., et al. 2007, ApJS, 171, 447

Rieke, G., Young, E., Engelbracht, C., et al. 2004, ApJS, 154, 25

Rodríguez, L., Anglada, G., \& Curiel, S. 1999, ApJS, 125, 427

Sadavoy, S., DiFrancesco, J., André, P., et al. 2014, ApJ, 787, 18

Sandell, G., \& Knee, L. 2001, ApJ, 546, 49

Scholz, A., Geers, V., Jayawardhana, R., et al. 2009, ApJ, 702, 805

Scholz, A., Jayawardhana, R., Muzic, K., et al. 2012a, ApJ, 756, 24

Scholz, A., Muzic, K., Geers, V., et al. 2012b, ApJ, 744, 6

Skrutskie, M., Cutri, R. M., Stiening, R., et al. 2006, AJ, 131, 1163

Walawender, J., Bally, J., Francesco, J., Joergensen, J., \& Getman, K. 2008, in Handbook of Star Forming Regions, Vol. 1, ed. B. Reipurth (San Francisco, CA: ASP), 346

Werner, M., Roellig, T., Low, F., et al. 2004, ApJS, 154, 1

Wilking, B., Meyer, M., Greene, T., Mikhail, A., \& Carlson, G. 2004, AJ, 127,1131

Wilking, B. A., Bontemps, S., Schuler, R. E., et al. 2001, ApJ, 551, 357

Winston, W., Megeath, S., Wolk, S., et al. 2009, AJ, 137, 4777

Winston, W., Megeath, S., Wolk, S., et al. 2010, AJ, 140, 266

Wright, E., Eisenhardt, P. R. M., Mainzer, A. K., et al. 2010, AJ, 140, 1868

Zasowski, G., Johnson, J. A., Frinchaboy, P. M., et al. 2013, AJ, 146, 81 